\title{
23. ORIGIN AND APPLICATIONS OF WHOLE-CORE MAGNETIC SUSCEPTIBILITY OF SEDIMENTS AND VOLCANIC ROCKS FROM LEG 152
}

\author{
Koji Fukuma
}

\begin{abstract}
Nearly continuous records of whole-core magnetic susceptibility obtained during Leg 152 reveal their potential as a lithostratigraphic tool for both sediments and volcanic rocks. For sediments, thermomagnetic and hysteresis measurements show that the whole-core magnetic susceptibility is primarily controlled by the concentration of magnetite associated with detrital minerals. The continuous susceptibility record for cores from Site 918 documents that the detrital influx gradually increased from about $11 \mathrm{Ma}$ and that already at about $9.5 \mathrm{Ma}$ the susceptibility reaches a high level about $50 \mathrm{~m}$ below the bottom of the oldest verified glaciomarine sediment (about $7 \mathrm{Ma}$ ). The two overlapping cores from Site 919 provide an opportunity for correlation and recovery of a complete composite sequence. In addition, the presence and type of dropstones can be detected by whole-core susceptibility if combined with the gamma-ray attenuation porosity evaluator (GRAPE) density data. For volcanic rocks, the whole-core susceptibility depends on the rock chemistry and the degree of oxidation. The susceptibility of the massive inner part of lava flows is lowest in the least evolved picrites and highest in the evolved olivine-free basalts in the Middle Series. Scoriaceous breccia and fractured rocks are characterized by highs of magnetic susceptibility. Although some of the lava flow tops are marked by susceptibility enhancement, very intense oxidation at other tops reduced the susceptibility by oxidizing magnetite into hematite. In situ susceptibility logging of subaerial volcanic sequences would be useful in taking a continuous profile of successions of lava flows. When combined with shore-based rock magnetic measurements, new applications of whole-core magnetic susceptibility data can be exploited to give new insights to lithostratigraphic variations.
\end{abstract}

\section{INTRODUCTION}

Since the installation of a whole-core magnetic susceptibility meter on the multisensor track on JOIDES Resolution, a great amount of susceptibility data has been accumulated. For sediments, the whole-core susceptibility measurements have provided several important paleoclimatic implications (e.g., Bloemendal and deMenocal, 1989; deMenocal et al., 1991); recently the susceptibility measurements were used as a rapid technique to estimate carbonate content, which in turn reflects paleoclimatic change (Leg 154 Scientific Party, 1995). Several useful applications, such as reconstructing complete sequences and detecting repeated sequences, have also been introduced by Robinson (1990). However, the application of whole-core magnetic susceptibility has yet to be fully exploited. The onboard measurement interval is frequently too rough to detect significant signals as a result of the limited time available for measurements. More seriously, the mechanism controlling susceptibility variation is not fully understood, and so it is difficult to give a valid interpretation of the susceptibility data.

Ocean Drilling Program (ODP) Leg 152 was designed to study the volcanic rifted margin off southeastern Greenland and the paleoceanographic events in the Irminger Basin (Larsen, Saunders, Clift, et al., 1994). Relatively continuous sequences of both sediments and volcanic rocks were recovered during Leg 152 . Whole-core magnetic susceptibility was measured at $2-\mathrm{cm}$ intervals for almost all of the cores of sediments and volcanic rocks. These measurements resulted in nearly continuous records of susceptibility variation, especially for the sediments recovered excellently by advanced hydraulic piston corer (APC) ( $100 \%$ recovery rates) and for the fully penetrated vol-

${ }^{1}$ Saunders, A.D., Larsen, H.C., and Wise, S.W., Jr. (Eds.), 1998. Proc. ODP, Sci. Results, 152: College Station, TX (Ocean Drilling Program).

${ }^{2}$ Rock Magnetism Laboratory, Department of Physics, Erindale College, University of Toronto, Mississauga, Ontario L5L 1C6, Canada. (Present address: Deep Sea Research Department, Japan Marine and Technology Center [JAMSTEC], 2-15 Natsushima-cho, Yokosuka 237, Japan.) fukuma@jamstec.go.jp canic basement at Site 917 (55.7\% of the $778.8-\mathrm{m}$ sequence was recovered). The high resolution data enabled me to analyze not only large-scale but also fine-scale (several-centimeter-scale) variations of the susceptibility data. Significant signals might be missed, or misleading interpretations drawn, however, by simply comparing susceptibility variations with the lithologic data. To determine the origin of the susceptibility variation, therefore, I determined the magnetic mineralogy and obtained hysteresis properties on discrete samples on shore.

\section{METHODS}

Magnetic susceptibility was measured on unsplit whole cores, using a Bartington Instruments' MS2C pass-through loop sensor (0.565 $\mathrm{kHz}, 0.1 \mathrm{mT}$ ) on the shipboard multisensor track. The measurement interval was set at $2 \mathrm{~cm}$ for most core sections, but it was set at $3 \mathrm{~cm}$ or more in some cases because of the limited time available for measurements. I converted raw reading values (in cgs units) to the volume-specific calibrated values (in SI units) using a paramagnetic calibration sample $\mathrm{MnO}\left(7.312 \times 10^{-6} \mathrm{G} / \mathrm{Oe}=9.184 \times 10^{-5} \mathrm{SI}\right)$ packed in a plastic core liner with the same diameter. Volcanic rocks do not fill core liners, so the calibrated susceptibility values should be regarded as minimum estimates. Much of the published ODP wholecore magnetic susceptibility data, including the Leg 152 Initial Reports (Larsen, Saunders, Clift, et al., 1994), however, are not calibrated and/or are shown in cgs units to avoid the accompanying confusion when converting from cgs to SI units (Moskowitz, 1995).

For sediment discrete samples, low-field susceptibility was measured on board and at the paleomagnetics laboratory of Kyoto University with a Bartington Instruments' susceptibility sensor MS2W in the low-frequency $(0.47 \mathrm{kHz})$ and high-frequency $(4.7 \mathrm{kHz})$ modes. Discrete samples were collected on board by pushing custom-made 9- $\mathrm{cm}^{3}$ plastic cubes into sediment cores. Because volume-specific susceptibility values were calculated by normalizing with the volume when the discrete samples still remained wet, it is possible to com- 
pare the values to the whole-core susceptibility values directly. Mass specific susceptibility values were taken on the basis of the mass measured after drying, so the values can be compared to the mass specific hysteresis parameters, which were obtained on dried small chips.

Thermomagnetic measurements were made with a horizontal $\mathrm{Cu}$ rie balance at Kyoto University by heating powder or small rock fragments in Ar gas or in air with an applied field of $0.4 \mathrm{~T}$, which is enough to saturate titanomagnetite and magnetite. Hysteresis loops were obtained on a few milligram samples using an alternating gradient force magnetometer (Princeton Measurement Cooperation "MicroMag") at the University of Toronto. Hysteresis parameters were calculated after subtracting para- and diamagnetic contributions.

\section{SEDIMENT RESULTS}

Figure 1 shows a downhole variation of magnetic susceptibility for the sedimentary sequences from Holes 918A and 918D in con-

\section{Holes 918A and 918D}
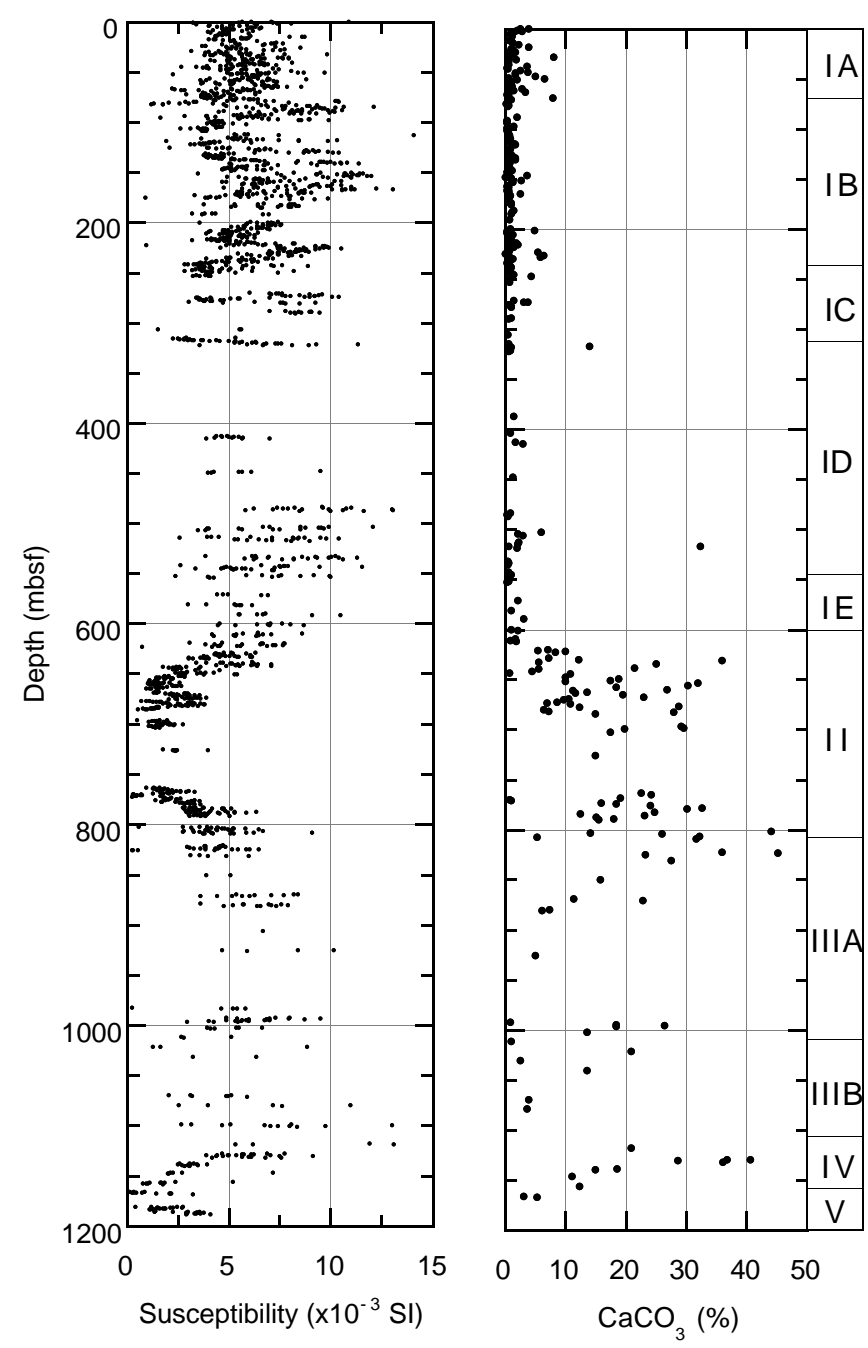

Figure 1. Downhole variation of whole-core magnetic susceptibility and calcium carbonate content of the sedimentary sequence from Holes 918A and 918D. Susceptibility data points are plotted at every 10th point of acquired data to avoid confusion. junction with the variation of calcium carbonate content. At Sites 914-917, the limited and discontinuous whole-core susceptibility data, due to the low core recovery rates, inhibited analyses of largescale features of susceptibility signals.

Instead of plotting too many points, the susceptibility data from Holes $918 \mathrm{~A}$ and $918 \mathrm{D}$ were plotted at every 10th data point (corresponds to every 20 - or 30-cm interval), which should be enough to reveal the large-scale features. The values and frequency are shown in comparison with the stratigraphic units (Larsen, Saunders, Clift, et al., 1994). Units IA-IIIE, IIIA-IIIB, and IV, which mainly comprise terrigenous or volcanogenic components, exhibit high values and a wide range (3-12 in $10^{-3} \mathrm{SI}$ ) associated with frequent changes, whereas Units II and V (mainly nannofossil chalk and glauconitic silt, respectively) have lower values (less than a few $10^{-3} \mathrm{SI}$ ) and infrequent variation. The magnetic susceptibility is inversely correlated with the calcium carbonate content. The reason is that the terrigenous and volcanogenic materials contain significant amounts of magnetic minerals, but biogenic carbonate is almost free of magnetic minerals. It is possible that variable early diagenesis, as suggested by the greenish gray color of the sediments and the possible presence of iron sulfide (see below), may be the cause of some part of the susceptibility variation. However, the gross feature of susceptibility variation can be primarily explained by the relative concentration of detrital (terrigenous and volcanogenic) vs. biogenic carbonate components.

The same explanation is applicable to the finer scale variation within Unit II as shown on Core 152-918D-39R (Fig. 2). The susceptibility change basically correlates with the alternating lithology of quartz silt with nannofossils and nannofossil chalk (Larsen, Saunders, Clift, et al., 1994) and two prominent peaks correspond to dark gray silt layers interbedded with greenish background sediments. It is evident that terrigenous or volcanogenic materials enhance magnetic susceptibility, and the susceptibility change is useful to detect such a change of the ratio of the detrital and biogenic components in a fine scale. The baseline values gradually increase upward in this core, and this trend can be found in larger scale in the upper part of Unit II (Fig. 1). Such a meso-scale feature probably also reflects the variation of detrital input relative to biogenic input, which is difficult to recognize in a continuous manner by other methods (e.g., visual core descriptions or calcium carbonate measurements). Based on the scattered data, Larsen et al. (1994) argued that a marked drop in carbonate content at 600 meters below seafloor (mbsf) ( 9.5 Ma) corresponded to climatic cooling; the lowest diamictite layer was identified (macroscopically) at $543.5 \mathrm{mbsf}$ (about $7 \mathrm{Ma}$ ). However, continuous magnetic susceptibility measurements suggest that despite the sudden drop in carbonate content, detrital input is gradually increasing upward from about $650 \mathrm{mbsf}(\sim 11 \mathrm{Ma})$.

If the whole-core susceptibility data are combined with GRAPE density data, we can infer rock types as well as positions of dropstones, which frequently occur in glaciomarine sediment cores, especially at the shelf sites (Sites 914-917). Figure 3 shows an example of susceptibility and GRAPE density variation related to dropstones. Whereas large increases in magnetic susceptibility correspond to mafic dropstones, nonmafic dropstones are marked by slight susceptibility increases or decreases depending on the lithology of the dropstone. Boulder-sized dropstones found at $3.35 \mathrm{mbsf}$ and $4.58 \mathrm{mbsf}$ give marked increases in GRAPE density, but a mafic dropstone at 4.13 mbsf, with a most prominent susceptibility peak in this interval, shows only a slight increase in GRAPE density due to its small size $(\sim 3 \mathrm{~cm})$. Prominent susceptibility peaks associated with even slight increases of GRAPE density mark the location of mafic dropstones. A susceptibility peak at 3.95 mbsf, which is associated with neither a peak in GRAPE density nor a visible dropstone, might represent a lithologic change. Peaks in GRAPE density but not in susceptibility values can be regarded as a mark for nonmafic dropstones. Small dropstones (less than a few centimeters) are difficult to detect by the combination of whole-core susceptibility and GRAPE density. These 


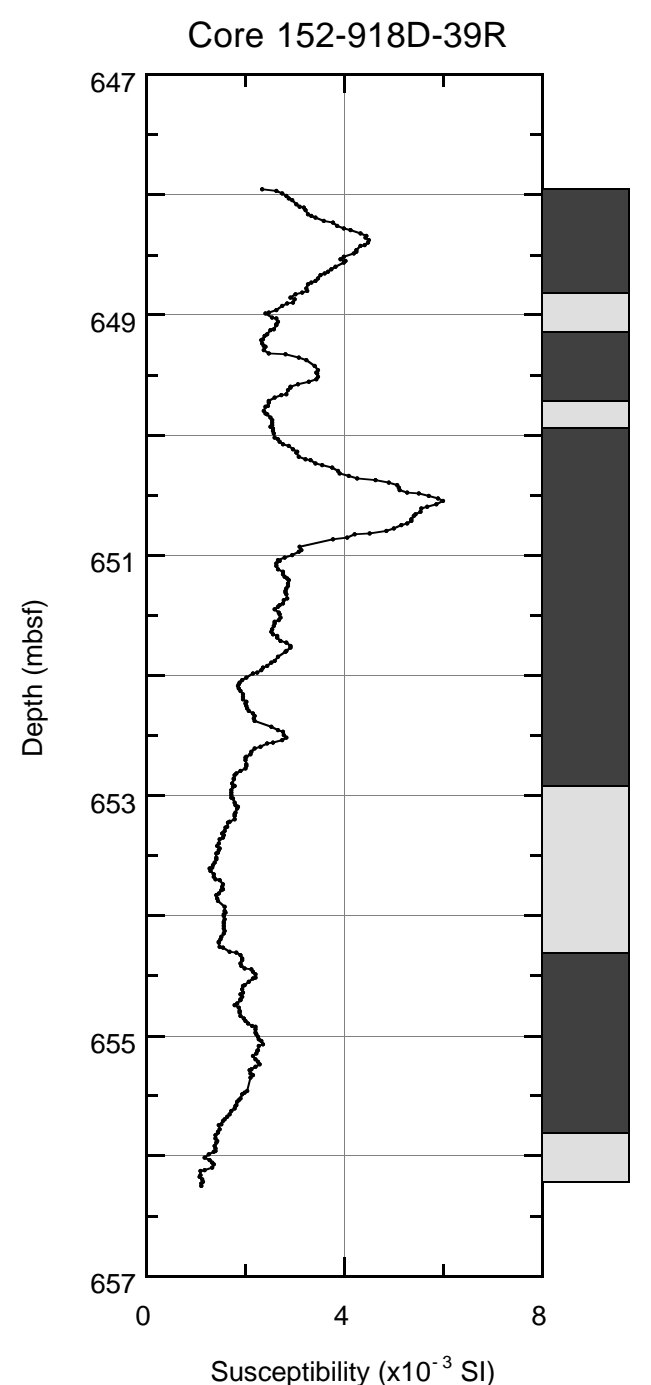

Quartz Silt with Nannofossils

Nannofossil Chalk

Figure 2. Whole-core magnetic susceptibility variation from Core 152-918D39R. Lithology is shown based on visual core descriptions (Larsen, Saunders, Clift, et al., 1994).

whole-core data were actually used when ODP technicians on board split cores to avoid disturbing matrix sediment by dropstones.

Dual APC cores were collected from overlapping sub-bottom depths down to about 20 mbsf from Holes 919A and 919B. By interhole correlation of whole-core magnetic susceptibility, these cores give an opportunity to detect the missing interval during coring and compare the sedimentation rates in detail. Dropstones at Site 919 occur infrequently and are small, unlike at the shelf sites (Sites 914917), making the interhole correlation much easier. The peak to peak correlation between Cores 919A-1H and 919B-1H is excellent, but the sedimentation rate is consistently about $10 \%$ higher at Hole 919B than it is at Hole 919A (Fig. 4). Although core recovery was very good at both holes (100.1\% for Hole 919A and $102.1 \%$ for Hole 919B), complete sequences were not necessarily recovered at these holes. Between $1 \mathrm{H}$ and $2 \mathrm{H}$ of both holes, several peaks were not correlated with each other. The missing interval probably amounts to 2 $\mathrm{m}$ between Cores 919B-1H and $2 \mathrm{H}$.

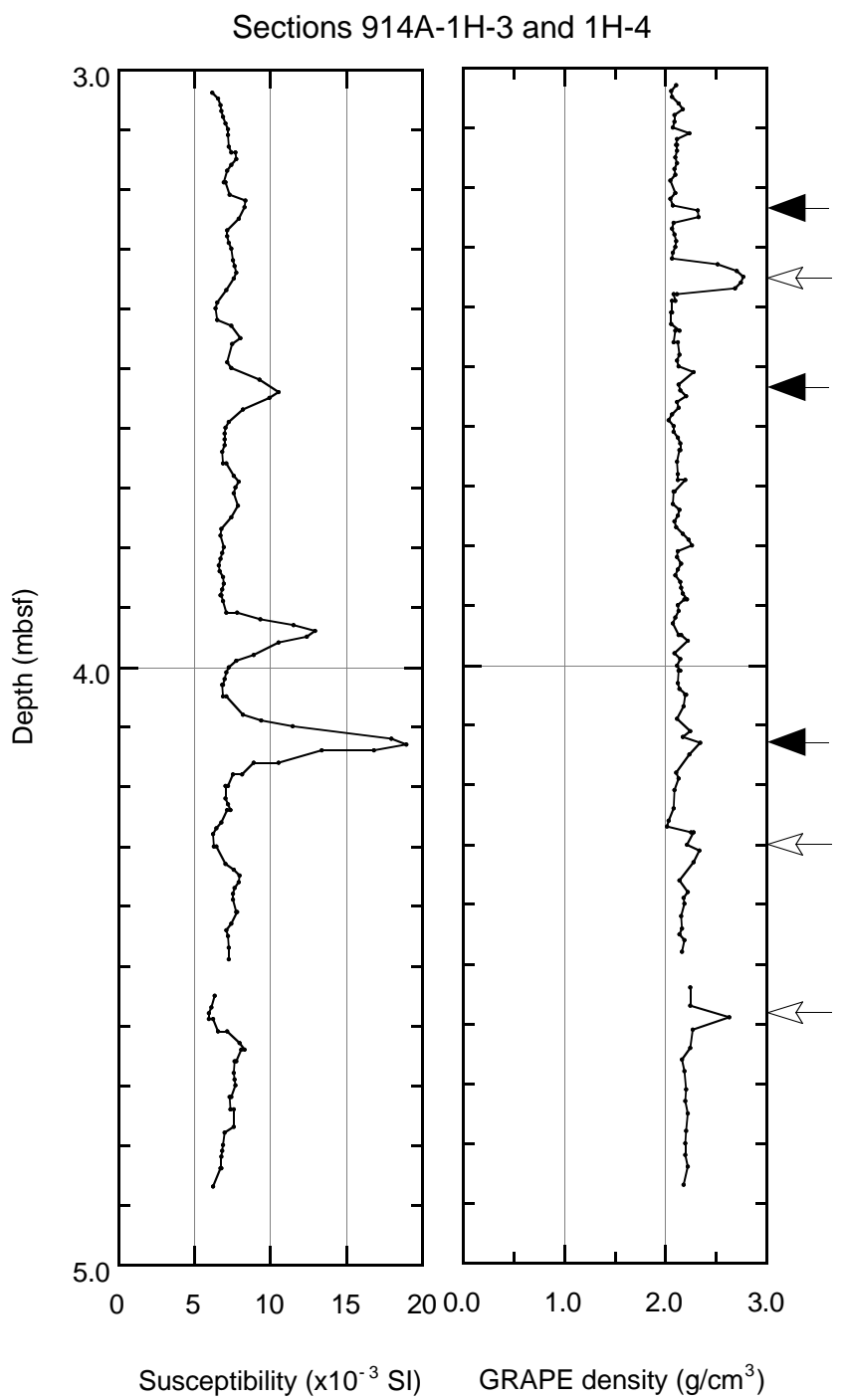

Figure 3. Whole-core magnetic susceptibility and GRAPE density variations for Sections 152-914A-1H-3 and 1H-4. Solid and open arrows indicate the position of mafic and nonmafic dropstones, respectively, confirmed by visual core descriptions (Larsen, Saunders, Clift, et al., 1994).

To identify the magnetic minerals of sediments recovered from Sites 918 and 919, I conducted thermomagnetic analyses in air and in an argon gas atmosphere. A typical example is shown in Figure 5. The heating curves are upward convex, suggesting that ferromagnetic minerals are dominant over paramagnetic minerals. A prominent kink on the heating curve is present at about $580^{\circ} \mathrm{C}$ of the Curie point of nearly pure magnetite, suggesting that the susceptibility signal mostly comes from nearly pure magnetite. There is no positive evidence for the presence of greigite or pyrrhotite in the thermomagnetic analyses; neither a significant decrease of magnetization between $270^{\circ}$ and $350^{\circ} \mathrm{C}$ expected for greigite (Roberts, 1995) nor pyrrhotite Curie point near $300^{\circ} \mathrm{C}$ (Schwartz, 1975) were seen in the thermomagnetic curves (Fig. 5). However, there are several characteristics suggesting the presence of minor iron sulfides, including paramagnetic pyrite. The magnetization increased above $400^{\circ} \mathrm{C}$ when heated in an argon gas, whereas no significant increase is seen when heated in air. Although the cooling curve is far below the heating curve under heating in air, the cooling curve is far above the heating curve in argon gas. Although these characteristics may be explained by oxida- 


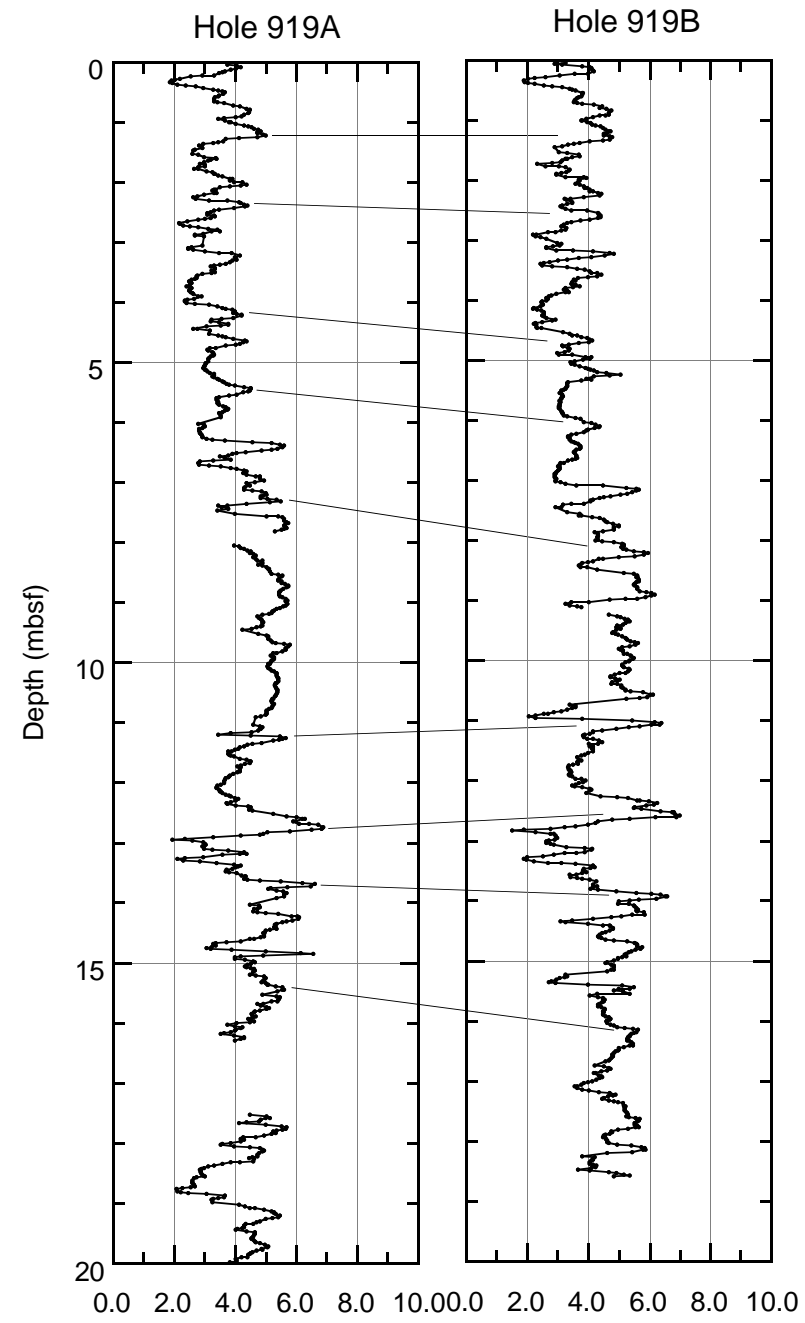

Susceptibility $\left(\times 10^{-3} \mathrm{SI}\right) \quad$ Susceptibility $\left(\times 10^{-3} \mathrm{SI}\right)$

Figure 4. Interhole correlation of whole-core magnetic susceptibility between Holes 919A and 919B. Some major peaks are correlated with lines between these two holes. At the core interval, data points are not tied by lines.

tion of iron-bearing silicates, it would be better to assume the presence of iron sulfides, taking into account the high magnetization intensity (saturation magnetization $=0.3 \sim 1.0 \mathrm{Am}^{2} / \mathrm{kg}$ ). Such iron sulfides would be a product of early reductive diagenesis (Karlin and Levi, 1983). A hump at about $130^{\circ} \mathrm{C}$ on the heating curves, which is particularly evident when heated in air (Fig. 5A), was found in all of the measured greenish gray samples (in reductive condition) but not found in the sample from a top brown 70-cm layer (in oxidative condition) at Site 919.

Table 1 lists hysteresis parameters on sediment discrete samples from Sites 918 and 919. For Site 918, hysteresis data were available only from Holes 918A, 918B, and 918C, which are restricted to the upper $320 \mathrm{~m}$ of lithologic Unit I. Some of these parameters (highfield mass susceptibility, saturation magnetization, and coercivity) are plotted against low-field volume susceptibility in Figure 6. Because low-field mass susceptibility, which can be directly compared to these mass specific hysteresis parameters, is well correlated to the low-field volume susceptibility, I plotted the hysteresis parameters vs. low-field volume susceptibility, which represents whole-core susceptibility. High-field mass susceptibility (on the order of $10^{-8} \mathrm{~m}^{3} /$
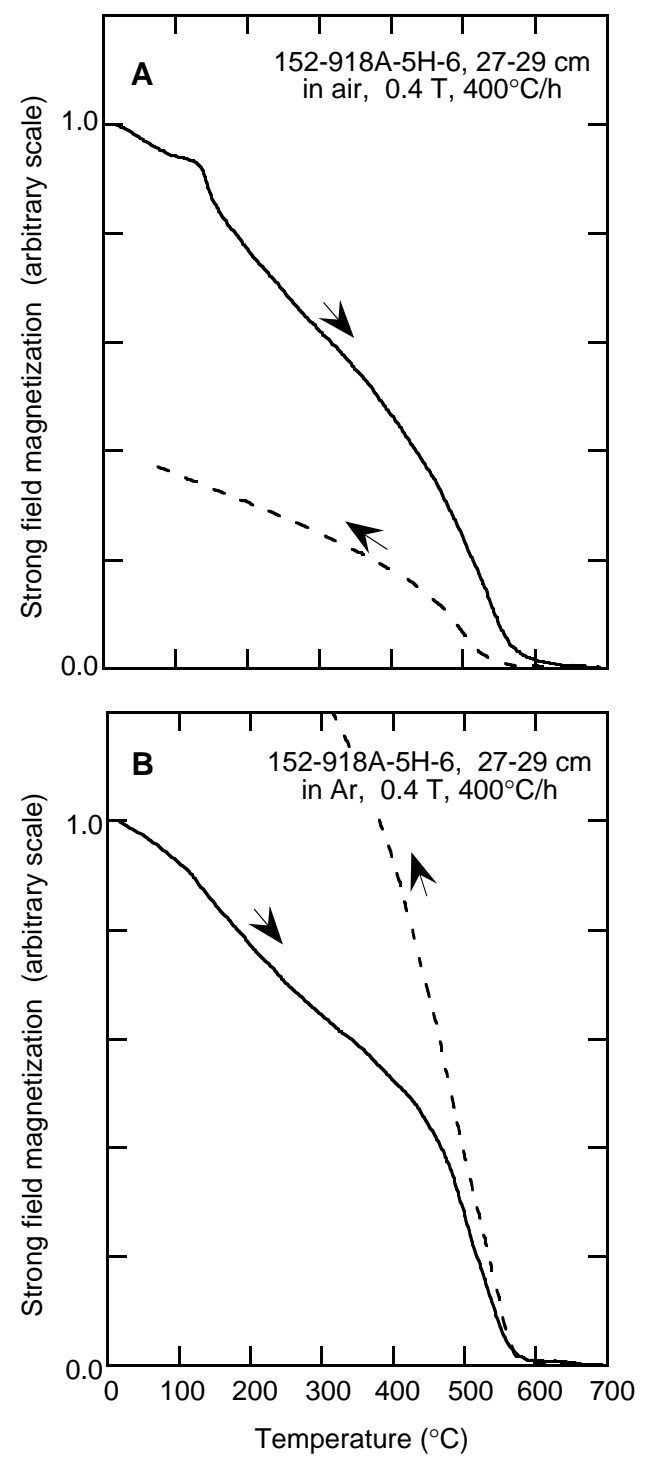

Figure 5. Representative thermomagnetic curves measured in air (A) and in an argon gas (B) for a sediment sequence from Site 918.

$\mathrm{kg}$ ), contributed by para- and diamagnetic minerals, is smaller by two orders of magnitude as compared with low-field mass susceptibility (Table 1). Some samples exhibit negative values, indicating dominant diamagnetic minerals (e.g., quartz or calcite). The correlation is very poor for both Sites 918 and 919, indicating that the whole-core susceptibility is not influenced by the concentration of para- and diamagnetic minerals (Fig. 6A, B). Figures 6C and 6D show the relationship between saturation magnetization of ferromagnetic minerals and low-field volume susceptibility. These two parameters correlate well with each other. Therefore, I can argue that the variation of the whole-core magnetic susceptibility reflects the concentration of ferromagnetic minerals. The grain size of ferromagnetic minerals may also contribute to the susceptibility change. To check the possibility, coercivity was plotted against volume susceptibility. There is no clear trend between these two parameters, as seen in Figures $6 \mathrm{E}$ and $6 \mathrm{~F}$. Except single domain/superparamagnetic threshold size, the grain size variation does not significantly affect the susceptibility variation (Maher, 1988; Heider et al., 1994). This finding justifies the use of whole-core magnetic susceptibility as a measure of the concentration of ferromagnetic minerals for Sites 918 and 919. 
Table 1. Susceptibility and hysteresis parameters on sediment discrete samples for Sites 918 and 919.

\begin{tabular}{|c|c|c|c|c|c|c|c|c|c|c|}
\hline $\begin{array}{l}\text { Core, section, } \\
\text { interval }(\mathrm{cm})\end{array}$ & $\begin{array}{l}\text { Depth } \\
\text { (mbsf) }\end{array}$ & $\begin{array}{l}\kappa_{\mathrm{lf}} \\
(\mathrm{SI})\end{array}$ & $\begin{array}{c}\chi_{\mathrm{lf}} \\
\left(\mathrm{m}^{3} / \mathrm{kg}\right)\end{array}$ & $\begin{array}{l}\kappa_{\mathrm{fd}} \\
(\%)\end{array}$ & $\begin{array}{c}\mathrm{J}_{\mathrm{s}} \\
\left(\mathrm{Am}^{2} / \mathrm{kg}\right)\end{array}$ & $\begin{array}{c}\mathrm{J}_{\mathrm{r}} \\
\left(\mathrm{Am}^{2} / \mathrm{kg}\right)\end{array}$ & $\begin{array}{l}\mathrm{H}_{\mathrm{c}} \\
(\mathrm{mT})\end{array}$ & $\begin{array}{c}\mathrm{H}_{\mathrm{cr}} \\
(\mathrm{mT})\end{array}$ & $\mathrm{J}_{\mathrm{r}} / \mathrm{J}_{\mathrm{S}}$ & $\mathrm{H}_{\mathrm{cr}} / \mathrm{H}_{\mathrm{c}}$ \\
\hline 152-918A- & & & & & & & & & & \\
\hline $5 \mathrm{H}-3,61-63$ & 33.91 & $2.81 \mathrm{e}-03$ & $2.65 e-06$ & 5.53 & $3.00 \mathrm{e}-01$ & $5.10 \mathrm{e}-02$ & 15.1 & 35.5 & 0.170 & 2.35 \\
\hline $5 \mathrm{H}-6,27-29$ & 38.07 & $6.12 \mathrm{e}-03$ & $6.14 \mathrm{e}-06$ & 2.18 & $7.53 \mathrm{e}-01$ & $1.60 \mathrm{e}-01$ & 16.5 & 36.2 & 0.212 & 2.19 \\
\hline $6 \mathrm{H}-2,46-48$ & 41.76 & $6.46 \mathrm{e}-03$ & $5.60 \mathrm{e}-06$ & 1.89 & $8.36 \mathrm{e}-01$ & $1.80 \mathrm{e}-01$ & 16.0 & 34.6 & 0.215 & 2.16 \\
\hline $6 \mathrm{H}-6,131-133$ & 48.61 & $4.32 \mathrm{e}-03$ & $3.89 \mathrm{e}-06$ & 2.57 & $5.04 \mathrm{e}-01$ & $7.77 e-02$ & 13.2 & 31.4 & 0.154 & 2.38 \\
\hline $7 \mathrm{H}-2,4-6$ & 50.84 & $6.32 \mathrm{e}-03$ & $6.09 \mathrm{e}-06$ & 2.64 & $9.27 \mathrm{e}-01$ & $2.15 \mathrm{e}-01$ & 18.2 & 35.9 & 0.232 & 1.97 \\
\hline $7 \mathrm{H}-4,98-100$ & 54.78 & $4.88 \mathrm{e}-03$ & $3.50 \mathrm{e}-06$ & 2.28 & $4.44 \mathrm{e}-01$ & $6.61 \mathrm{e}-02$ & 13.7 & 33.7 & 0.149 & 2.46 \\
\hline $8 \mathrm{H}-2,89-91$ & 61.19 & $3.21 \mathrm{e}-03$ & $2.82 \mathrm{e}-06$ & 3.11 & $3.24 \mathrm{e}-01$ & $5.26 \mathrm{e}-02$ & 13.8 & 32.2 & 0.162 & 2.33 \\
\hline $8 \mathrm{H}-5,103-105$ & 65.83 & $6.06 \mathrm{e}-03$ & $4.40 \mathrm{e}-06$ & 3.12 & $5.13 \mathrm{e}-01$ & $9.31 \mathrm{e}-02$ & 15.4 & 35.5 & 0.181 & 2.31 \\
\hline $9 \mathrm{H}-3,56-58$ & 71.86 & $3.84 \mathrm{e}-03$ & $3.04 \mathrm{e}-06$ & 2.02 & $3.86 \mathrm{e}-01$ & $5.37 \mathrm{e}-02$ & 12.8 & 33.5 & 0.139 & 2.62 \\
\hline $9 \mathrm{H}-6,91-93$ & 76.71 & $4.90 \mathrm{e}-03$ & $3.73 e-06$ & 2.72 & $4.21 \mathrm{e}-01$ & $6.98 \mathrm{e}-02$ & 15.1 & 35.9 & 0.166 & 2.38 \\
\hline $10 \mathrm{H}-2,70-72$ & 80.00 & $1.04 \mathrm{e}-02$ & $6.56 \mathrm{e}-06$ & 1.81 & $9.33 \mathrm{e}-01$ & $2.01 \mathrm{e}-01$ & 16.4 & 35.8 & 0.215 & 2.18 \\
\hline $10 \mathrm{H}-6,40-42$ & 85.70 & $8.41 \mathrm{e}-03$ & $6.06 e-06$ & 2.64 & $1.04 \mathrm{e}+00$ & $2.24 \mathrm{e}-01$ & 16.0 & 34.8 & 0.215 & 2.17 \\
\hline $11 \mathrm{H}-2,96-98$ & 89.76 & $8.11 \mathrm{e}-04$ & $5.42 \mathrm{e}-07$ & 0.00 & $1.67 \mathrm{e}-02$ & $2.30 \mathrm{e}-03$ & 12.6 & 31.9 & 0.138 & 2.53 \\
\hline $11 \mathrm{H}-6,40-42$ & 95.20 & $5.74 \mathrm{e}-03$ & $4.56 \mathrm{e}-06$ & 2.71 & $8.26 \mathrm{e}-01$ & $1.87 \mathrm{e}-01$ & 17.1 & 37.7 & 0.226 & 2.20 \\
\hline $12 \mathrm{H}-2,102-104$ & 99.32 & $3.63 \mathrm{e}-03$ & $2.77 \mathrm{e}-06$ & 2.75 & $3.41 \mathrm{e}-01$ & $5.10 \mathrm{e}-02$ & 13.8 & 33.6 & 0.150 & 2.43 \\
\hline $12 \mathrm{H}-6,20-22$ & 104.50 & $3.88 \mathrm{e}-03$ & $2.73 e-06$ & 3.44 & $3.86 \mathrm{e}-01$ & $5.05 e-02$ & 13.5 & 35.3 & 0.131 & 2.61 \\
\hline $13 \mathrm{H}-2,70-72$ & 108.50 & $3.94 \mathrm{e}-03$ & $2.76 \mathrm{e}-06$ & 2.82 & $3.99 \mathrm{e}-01$ & $5.94 \mathrm{e}-02$ & 13.6 & 32.4 & 0.149 & 2.38 \\
\hline $13 \mathrm{H}-4,129-131$ & 112.09 & $3.88 \mathrm{e}-03$ & $3.02 \mathrm{e}-06$ & 3.15 & $4.24 \mathrm{e}-01$ & $6.98 \mathrm{e}-02$ & 14.3 & 33.6 & 0.165 & 2.35 \\
\hline $14 \mathrm{H}-2,80-82$ & 118.10 & $6.00 \mathrm{e}-03$ & $4.37 \mathrm{e}-06$ & 7.05 & $5.81 \mathrm{e}-01$ & $1.22 \mathrm{e}-01$ & 16.2 & 36.1 & 0.210 & 2.23 \\
\hline $14 \mathrm{H}-5,41-43$ & 122.21 & $3.43 e-03$ & $2.61 \mathrm{e}-06$ & 3.88 & $3.44 \mathrm{e}-01$ & $5.42 \mathrm{e}-02$ & 14.0 & 31.0 & 0.158 & 2.21 \\
\hline $15 \mathrm{H}-2,80-82$ & 127.60 & $6.64 \mathrm{e}-03$ & $4.27 \mathrm{e}-06$ & 1.84 & $5.79 \mathrm{e}-01$ & $8.87 e-02$ & 13.7 & 33.9 & 0.153 & 2.47 \\
\hline $15 \mathrm{H}-4,80-82$ & 130.60 & $9.51 \mathrm{e}-03$ & $5.97 \mathrm{e}-06$ & 2.34 & $8.50 \mathrm{e}-01$ & $1.70 \mathrm{e}-01$ & 15.7 & 36.5 & 0.200 & 2.32 \\
\hline $16 \mathrm{H}-2,48-50$ & 135.28 & $3.42 \mathrm{e}-03$ & & 0.97 & & & & & & \\
\hline $16 \mathrm{H}-5,4-6$ & 139.34 & $7.67 \mathrm{e}-03$ & $6.07 \mathrm{e}-06$ & 2.75 & $8.77 \mathrm{e}-01$ & $2.04 \mathrm{e}-01$ & 17.5 & 38.9 & 0.233 & 2.22 \\
\hline $17 \mathrm{H}-1,84-86$ & 143.64 & $6.02 \mathrm{e}-03$ & $4.53 e-06$ & 2.58 & $5.71 \mathrm{e}-01$ & $1.41 \mathrm{e}-01$ & 18.1 & 39.3 & 0.247 & 2.17 \\
\hline $17 \mathrm{H}-4,57-59$ & 147.87 & $4.50 \mathrm{e}-03$ & $3.18 \mathrm{e}-06$ & 2.47 & $2.75 \mathrm{e}-01$ & $6.60 \mathrm{e}-02$ & 17.9 & 38.9 & 0.240 & 2.17 \\
\hline $18 \mathrm{H}-2,125-127$ & 155.05 & $5.40 \mathrm{e}-03$ & $3.86 \mathrm{e}-06$ & 2.06 & $5.19 \mathrm{e}-01$ & $1.06 \mathrm{e}-01$ & 15.8 & 34.6 & 0.204 & 2.19 \\
\hline $18 \mathrm{H}-5,61-63$ & 158.91 & $1.04 \mathrm{e}-02$ & $6.52 \mathrm{e}-06$ & 2.03 & $9.61 \mathrm{e}-01$ & $1.86 \mathrm{e}-01$ & 15.0 & 34.3 & 0.194 & 2.29 \\
\hline $19 \mathrm{H}-1,130-132$ & 163.10 & $5.31 \mathrm{e}-03$ & $3.66 \mathrm{e}-06$ & 2.93 & $5.46 \mathrm{e}-01$ & $1.16 \mathrm{e}-01$ & 15.8 & 35.4 & 0.212 & 2.24 \\
\hline $19 \mathrm{H}-4,104-106$ & 167.34 & $8.50 \mathrm{e}-03$ & $6.71 \mathrm{e}-06$ & 2.75 & $8.48 \mathrm{e}-01$ & $1.94 \mathrm{e}-01$ & 17.3 & 38.0 & 0.229 & 2.20 \\
\hline $20 X-4,44-46$ & 176.24 & $9.29 \mathrm{e}-03$ & $5.82 \mathrm{e}-06$ & 2.15 & $9.14 \mathrm{e}-01$ & $1.73 e-01$ & 15.1 & 35.9 & 0.189 & 2.38 \\
\hline $21 \mathrm{X}-2,128-130$ & 184.28 & $8.36 \mathrm{e}-03$ & $5.29 \mathrm{e}-06$ & 1.46 & $8.97 \mathrm{e}-01$ & $1.26 \mathrm{e}-01$ & 13.1 & 36.0 & 0.140 & 2.75 \\
\hline $23 X-3,90-92$ & 203.70 & $5.94 \mathrm{e}-03$ & $5.11 \mathrm{e}-06$ & 2.43 & $8.58 \mathrm{e}-01$ & $2.07 \mathrm{e}-01$ & 17.1 & 39.0 & 0.241 & 2.28 \\
\hline $23 X-5,44-46$ & 206.24 & $6.61 e-03$ & $4.48 \mathrm{e}-06$ & 1.85 & $6.20 \mathrm{e}-01$ & $1.49 \mathrm{e}-01$ & 17.8 & 41.0 & 0.240 & 2.30 \\
\hline $24 X-2,83-85$ & 211.03 & $5.08 \mathrm{e}-03$ & $3.49 \mathrm{e}-06$ & 2.63 & $4.16 \mathrm{e}-01$ & $9.67 e-02$ & 16.7 & 38.8 & 0.232 & 2.32 \\
\hline $24 X-6,58-60$ & 216.78 & $4.06 e-03$ & $2.75 e-06$ & 2.19 & $4.69 \mathrm{e}-01$ & $1.06 \mathrm{e}-01$ & 17.3 & 41.4 & 0.226 & 2.39 \\
\hline $25 X-2,68-70$ & 219.78 & $5.40 \mathrm{e}-03$ & $4.11 \mathrm{e}-06$ & 2.26 & $5.49 \mathrm{e}-01$ & $1.19 \mathrm{e}-01$ & 16.3 & 36.5 & 0.217 & 2.24 \\
\hline $25 X-6,70-72$ & 225.80 & $5.93 \mathrm{e}-03$ & $4.62 \mathrm{e}-06$ & 2.81 & $6.69 \mathrm{e}-01$ & $1.61 \mathrm{e}-01$ & 18.2 & 40.5 & 0.241 & 2.23 \\
\hline $26 \mathrm{X}-2,120-122$ & 229.20 & $7.56 e-03$ & $6.14 \mathrm{e}-06$ & 2.35 & $7.99 \mathrm{e}-01$ & $1.87 \mathrm{e}-01$ & 17.3 & 37.6 & 0.234 & 2.17 \\
\hline $26 X-5,79-81$ & 233.29 & $8.00 \mathrm{e}-03$ & $6.19 \mathrm{e}-06$ & 2.09 & $9.55 \mathrm{e}-01$ & $2.11 \mathrm{e}-01$ & 16.6 & 36.9 & 0.221 & 2.22 \\
\hline $27 X-2,58-60$ & 237.28 & $6.60 \mathrm{e}-03$ & $4.92 \mathrm{e}-06$ & 2.69 & $6.39 \mathrm{e}-01$ & $1.51 \mathrm{e}-01$ & 17.8 & 38.9 & 0.236 & 2.19 \\
\hline $27 X-4,87-89$ & 240.57 & $3.62 \mathrm{e}-03$ & $2.85 \mathrm{e}-06$ & 1.84 & $4.19 \mathrm{e}-01$ & $9.76 \mathrm{e}-02$ & 17.9 & 41.4 & 0.233 & 2.31 \\
\hline $28 X-2,49-51$ & 246.09 & $5.72 \mathrm{e}-03$ & $4.42 \mathrm{e}-06$ & 2.91 & $7.05 \mathrm{e}-01$ & $1.58 \mathrm{e}-01$ & 17.1 & 39.6 & 0.224 & 2.32 \\
\hline $28 X-4,6-8$ & 248.66 & $1.84 \mathrm{e}-03$ & $1.23 \mathrm{e}-06$ & 0.60 & $4.38 \mathrm{e}-01$ & $3.35 \mathrm{e}-02$ & 6.9 & 27.2 & 0.0765 & 3.91 \\
\hline $31 X-1,91-93$ & 271.51 & $6.24 \mathrm{e}-03$ & $5.78 \mathrm{e}-06$ & 1.96 & $8.43 e-01$ & $1.75 e-01$ & 16.1 & 36.0 & 0.208 & 2.23 \\
\hline $31 X-4,56-58$ & 275.66 & $3.24 \mathrm{e}-03$ & $2.72 \mathrm{e}-06$ & 1.03 & $4.27 \mathrm{e}-01$ & $5.54 \mathrm{e}-02$ & 12.9 & 33.0 & 0.130 & 2.56 \\
\hline $33 \mathrm{X}-1,98-100$ & 289.38 & $8.16 \mathrm{e}-03$ & $6.60 \mathrm{e}-06$ & 2.86 & $1.03 e+00$ & $2.29 \mathrm{e}-01$ & 16.6 & 35.6 & 0.222 & 2.14 \\
\hline $37 \mathrm{X}-2,107-109$ & 317.67 & $3.82 \mathrm{e}-03$ & $2.61 \mathrm{e}-06$ & 1.74 & $3.46 \mathrm{e}-01$ & $7.35 e-02$ & 16.0 & 35.2 & 0.212 & 2.20 \\
\hline $37 X-4,133-135$ & 320.93 & $7.40 \mathrm{e}-03$ & $4.93 e-06$ & 2.10 & $8.20 \mathrm{e}-01$ & $1.45 \mathrm{e}-01$ & 14.7 & 34.2 & 0.177 & 2.33 \\
\hline 152-918B- & & & & & & & & & & \\
\hline $1-43$ & 0.41 & $3.20 \mathrm{e}-03$ & & 2.08 & & & & & & \\
\hline $1 \mathrm{H}-1,77-79$ & 0.77 & $2.72 \mathrm{e}-03$ & $2.71 \mathrm{e}-06$ & 3.67 & $4.15 \mathrm{e}-01$ & $7.56 e-02$ & 15.0 & 35.2 & 0.182 & 2.35 \\
\hline $1 \mathrm{H}-4,52-54$ & 5.02 & $3.79 \mathrm{e}-03$ & $3.17 \mathrm{e}-06$ & 3.23 & $4.57 \mathrm{e}-01$ & $7.97 e-02$ & 13.8 & 33.1 & 0.174 & 2.40 \\
\hline $1 \mathrm{H}-4,82-84$ & 5.32 & $8.03 e-03$ & & 1.38 & & & & & & \\
\hline $1 \mathrm{H}-5,72-74$ & 6.72 & $3.83 \mathrm{e}-03$ & $3.28 \mathrm{e}-06$ & 2.90 & $4.70 \mathrm{e}-01$ & $8.52 \mathrm{e}-02$ & 15.2 & 35.5 & 0.181 & 2.34 \\
\hline $2 \mathrm{H}-2,93-95$ & 9.23 & $6.20 \mathrm{e}-03$ & $3.48 \mathrm{e}-06$ & 1.61 & $5.85 \mathrm{e}-01$ & $1.01 \mathrm{e}-01$ & 14.3 & 35.6 & 0.173 & 2.49 \\
\hline $2 \mathrm{H}-3,64-66$ & 10.44 & $3.29 \mathrm{e}-03$ & $2.66 \mathrm{e}-06$ & 4.05 & $3.52 \mathrm{e}-01$ & $5.67 \mathrm{e}-02$ & 14.2 & 35.5 & 0.161 & 2.50 \\
\hline $2 \mathrm{H}-5,38-40$ & 13.18 & $1.03 \mathrm{e}-02$ & $6.09 \mathrm{e}-06$ & 1.19 & $9.55 \mathrm{e}-01$ & $1.34 \mathrm{e}-01$ & 12.1 & 33.9 & 0.140 & 2.80 \\
\hline $2 \mathrm{H}-5,118-120$ & 13.98 & $4.29 \mathrm{e}-03$ & $3.49 e-06$ & 3.37 & $5.27 \mathrm{e}-01$ & $9.66 \mathrm{e}-02$ & 14.8 & 35.1 & 0.183 & 2.37 \\
\hline $2 \mathrm{H}-6,89-91$ & 15.19 & $4.92 \mathrm{e}-03$ & $2.98 \mathrm{e}-06$ & 1.35 & $3.31 \mathrm{e}-01$ & $3.64 \mathrm{e}-02$ & 10.1 & 31.3 & 0.110 & 3.10 \\
\hline $3 \mathrm{H}-2,36-38$ & 18.16 & $5.99 \mathrm{e}-03$ & $4.46 e-06$ & 2.04 & $8.09 \mathrm{e}-01$ & $1.36 \mathrm{e}-01$ & 14.7 & 36.7 & 0.168 & 2.50 \\
\hline $3 \mathrm{H}-2,109-111$ & 18.89 & $2.43 e-03$ & $2.16 \mathrm{e}-06$ & 1.37 & $3.62 \mathrm{e}-01$ & $7.66 e-02$ & 16.9 & 37.8 & 0.212 & 2.24 \\
\hline $3 \mathrm{H}-4,65-67$ & 21.45 & $3.97 \mathrm{e}-03$ & $2.99 \mathrm{e}-06$ & 5.04 & $4.16 \mathrm{e}-01$ & $6.51 \mathrm{e}-02$ & 14.8 & 37.6 & 0.156 & 2.54 \\
\hline $3 \mathrm{H}-5,30-32$ & 22.60 & $7.78 e-03$ & $4.91 \mathrm{e}-06$ & 1.29 & $7.09 \mathrm{e}-01$ & $9.46 e-02$ & 11.0 & 27.9 & 0.133 & 2.54 \\
\hline $3 \mathrm{H}-5,127-129$ & 23.57 & $3.77 \mathrm{e}-03$ & $2.82 \mathrm{e}-06$ & 4.13 & $4.06 \mathrm{e}-01$ & $6.22 \mathrm{e}-02$ & 13.6 & 33.9 & 0.153 & 2.49 \\
\hline 28 & & & & & & & & & & \\
\hline $32-34$ & 29.12 & $5.78 \mathrm{e}-03$ & $4.90 \mathrm{e}-06$ & 2.50 & $1.03 \mathrm{e}-01$ & $1.67 \mathrm{e}-02$ & 14.2 & 37.7 & 0.162 & 2.65 \\
\hline $1 \mathrm{H}-3,113-115$ & 29.93 & $3.17 \mathrm{e}-03$ & $2.25 \mathrm{e}-06$ & 4.56 & $3.05 \mathrm{e}-01$ & $5.24 \mathrm{e}-02$ & 14.8 & 34.3 & 0.172 & 2.32 \\
\hline $1 \mathrm{H}-3,134-136$ & 30.14 & $4.68 \mathrm{e}-03$ & $3.36 \mathrm{e}-06$ & 2.14 & $6.07 \mathrm{e}-01$ & $1.12 \mathrm{e}-01$ & 14.8 & 35.0 & 0.185 & 2.36 \\
\hline $1 \mathrm{H}-5,45-47$ & 32.25 & $3.46 \mathrm{e}-03$ & $2.31 \mathrm{e}-06$ & 1.61 & $3.12 \mathrm{e}-01$ & $5.23 \mathrm{e}-02$ & 14.9 & 34.7 & 0.168 & 2.33 \\
\hline $1 \mathrm{H}-6,60-62$ & 33.90 & $4.23 \mathrm{e}-03$ & $2.66 \mathrm{e}-06$ & 2.10 & $3.48 \mathrm{e}-01$ & $4.76 \mathrm{e}-02$ & 12.7 & 33.2 & 0.137 & 2.61 \\
\hline $1 \mathrm{H}-6,78-80$ & 34.08 & $9.03 e-03$ & $5.18 \mathrm{e}-06$ & 1.48 & $8.28 \mathrm{e}-01$ & $1.02 \mathrm{e}-01$ & 12.1 & 33.1 & 0.123 & 2.74 \\
\hline 152-919A- & & & & & & & & & & \\
\hline $1 \mathrm{H}-1,44-46$ & 0.44 & $3.53 e-03$ & $1.87 \mathrm{e}-06$ & 2.2 & 01 & $1.13 \mathrm{e}-$ & 17 & 45 & 0.208 & 2.62 \\
\hline $1 \mathrm{H}-2,54-56$ & 2.04 & $3.92 \mathrm{e}-03$ & $3.92 \mathrm{e}-06$ & 3.12 & $6.73 \mathrm{e}-01$ & $1.55 \mathrm{e}-01$ & 19. & 48.8 & 0.230 & 2.55 \\
\hline $1 \mathrm{H}-4,48-50$ & 4.98 & $3.00 \mathrm{e}-03$ & $3.37 \mathrm{e}-06$ & 4.44 & $4.42 \mathrm{e}-01$ & $8.33 \mathrm{e}-02$ & 16.1 & 36.7 & 0.188 & 2.28 \\
\hline $2 \mathrm{H}-3,80-82$ & 11.80 & $4.03 e-03$ & $3.85 e-06$ & 2.75 & $4.93 e-01$ & $1.11 \mathrm{e}-01$ & 17.4 & 39.2 & 0.225 & 2.25 \\
\hline $2 \mathrm{H}-5,84-86$ & 14.84 & $4.30 \mathrm{e}-03$ & $4.14 \mathrm{e}-06$ & 3.10 & $7.65 \mathrm{e}-01$ & $1.69 \mathrm{e}-01$ & 17.6 & 38.7 & 0.221 & 2.20 \\
\hline $3 \mathrm{H}-1,71-73$ & 18.21 & $4.38 \mathrm{e}-03$ & $3.61 \mathrm{e}-06$ & 1.52 & $6.40 \mathrm{e}-01$ & $1.26 \mathrm{e}-01$ & 16.1 & 35.0 & 0.197 & 2.17 \\
\hline $3 \mathrm{H}-3,92-94$ & 21.42 & $3.07 \mathrm{e}-03$ & $2.40 \mathrm{e}-06$ & 2.54 & $3.11 \mathrm{e}-01$ & $5.17 \mathrm{e}-02$ & 15.0 & 34.9 & 0.166 & 2.33 \\
\hline $3 \mathrm{H}-5,110-112$ & 24.60 & $5.14 \mathrm{e}-03$ & $5.14 \mathrm{e}-06$ & 2.38 & $8.36 \mathrm{e}-01$ & $1.83 \mathrm{e}-01$ & 16.6 & 36.6 & 0.219 & 2.20 \\
\hline $3 \mathrm{H}-6,11-13$ & 25.11 & $4.73 e-03$ & $5.38 \mathrm{e}-06$ & 2.35 & $7.96 \mathrm{e}-01$ & $1.74 \mathrm{e}-01$ & 17.0 & 36.9 & 0.219 & 2.17 \\
\hline $4 \mathrm{H}-2,59-61$ & 29.09 & $3.43 e-03$ & $4.27 \mathrm{e}-06$ & 2.59 & $7.51 \mathrm{e}-01$ & $1.63 \mathrm{e}-01$ & 16.5 & 36.0 & 0.217 & 2.18 \\
\hline $4 \mathrm{H}-3,58-60$ & 30.58 & $7.94 \mathrm{e}-03$ & $5.73 e-06$ & 1.82 & $9.69 \mathrm{e}-01$ & $1.64 \mathrm{e}-01$ & 14.4 & 35.6 & 0.169 & 2.47 \\
\hline $4 \mathrm{H}-5,19-21$ & 33.19 & $4.92 \mathrm{e}-03$ & $4.55 \mathrm{e}-06$ & 3.61 & $5.64 \mathrm{e}-01$ & $1.33 \mathrm{e}-01$ & 17.7 & 38.1 & 0.236 & 2.15 \\
\hline $4 \mathrm{H}-6,21-23$ & 34.71 & $3.11 \mathrm{e}-03$ & $2.55 \mathrm{e}-06$ & 5.00 & $2.86 \mathrm{e}-01$ & $4.90 \mathrm{e}-02$ & 15.5 & 35.4 & 0.171 & 2.28 \\
\hline
\end{tabular}


Table 1 (continued).

\begin{tabular}{|c|c|c|c|c|c|c|c|c|c|c|c|}
\hline $\begin{array}{l}\text { Core, section, } \\
\text { interval }(\mathrm{cm})\end{array}$ & $\begin{array}{l}\text { Depth } \\
\text { (mbsf) }\end{array}$ & $\begin{array}{c}\kappa_{\mathrm{lf}} \\
(\mathrm{SI})\end{array}$ & $\begin{array}{c}\chi_{\mathrm{lf}} \\
\left(\mathrm{m}^{3} / \mathrm{kg}\right)\end{array}$ & $\begin{array}{c}\kappa_{\mathrm{fd}} \\
(\%)\end{array}$ & $\begin{array}{c}\mathrm{J}_{\mathrm{s}} \\
\left(\mathrm{Am}^{2} / \mathrm{kg}\right)\end{array}$ & $\begin{array}{c}\mathrm{J}_{\mathrm{r}} \\
\left(\mathrm{Am}^{2} / \mathrm{kg}\right)\end{array}$ & $\begin{array}{c}\mathrm{H}_{\mathrm{c}} \\
(\mathrm{mT})\end{array}$ & $\begin{array}{l}\mathrm{H}_{\mathrm{cr}} \\
(\mathrm{mT})\end{array}$ & $\mathrm{J}_{\mathrm{r}} / \mathrm{J}_{\mathrm{s}}$ & $\mathrm{H}_{\mathrm{cr}} / \mathrm{H}_{\mathrm{c}}$ & $\begin{array}{c}\chi_{\mathrm{hf}} \\
\left(\mathrm{m}^{3} / \mathrm{kg}\right)\end{array}$ \\
\hline $5 \mathrm{H}-2,44-46$ & 38.44 & $5.07 \mathrm{e}-03$ & & 2.63 & & & & & & & \\
\hline $5 \mathrm{H}-3,68-70$ & 40.18 & $2.76 \mathrm{e}-03$ & $3.02 \mathrm{e}-06$ & 3.63 & $3.30 \mathrm{e}-01$ & $6.34 \mathrm{e}-02$ & 15.1 & 34.6 & 0.192 & 2.29 & $-7.13 \mathrm{e}-09$ \\
\hline $5 \mathrm{H}-5,85-87$ & 43.35 & $3.80 \mathrm{e}-03$ & $4.55 \mathrm{e}-06$ & 3.22 & $7.41 \mathrm{e}-01$ & $1.82 \mathrm{e}-01$ & 19.0 & 40.9 & 0.246 & 2.15 & $1.65 \mathrm{e}-07$ \\
\hline $5 \mathrm{H}-6,41-43$ & 44.41 & $7.13 e-03$ & $6.03 \mathrm{e}-06$ & 2.18 & $9.55 \mathrm{e}-01$ & $1.92 \mathrm{e}-01$ & 16.2 & 36.2 & 0.201 & 2.23 & $1.43 \mathrm{e}-07$ \\
\hline $6 \mathrm{H}-1,120-122$ & 47.20 & $5.68 \mathrm{e}-03$ & $5.39 \mathrm{e}-06$ & 2.94 & $9.24 \mathrm{e}-01$ & $1.92 \mathrm{e}-01$ & 16.4 & 36.0 & 0.208 & 2.20 & $4.94 \mathrm{e}-08$ \\
\hline $6 \mathrm{H}-3,140-142$ & 50.40 & $7.73 e-03$ & $6.24 \mathrm{e}-06$ & 2.01 & $9.52 \mathrm{e}-01$ & $1.73 \mathrm{e}-01$ & 14.5 & 32.9 & 0.182 & 2.27 & $8.63 e-08$ \\
\hline $6 \mathrm{H}-4,140-142$ & 51.90 & $6.79 \mathrm{e}-03$ & $5.98 \mathrm{e}-06$ & 3.60 & $7.54 \mathrm{e}-01$ & $1.76 \mathrm{e}-01$ & 18.0 & 39.9 & 0.233 & 2.22 & $2.90 \mathrm{e}-08$ \\
\hline $6 \mathrm{H}-5,125-127$ & 53.25 & $3.81 \mathrm{e}-03$ & $3.49 \mathrm{e}-06$ & 2.62 & $3.98 \mathrm{e}-01$ & $9.49 \mathrm{e}-02$ & 18.8 & 39.3 & 0.238 & 2.09 & $4.47 \mathrm{e}-08$ \\
\hline $7 \mathrm{H}-3,63-65$ & 59.13 & $7.40 \mathrm{e}-03$ & $5.30 \mathrm{e}-06$ & 2.40 & $9.14 \mathrm{e}-01$ & $1.94 \mathrm{e}-01$ & 16.1 & 34.9 & 0.212 & 2.17 & $1.26 \mathrm{e}-07$ \\
\hline $7 \mathrm{H}-4,78-80$ & 60.78 & $7.80 \mathrm{e}-03$ & $6.01 \mathrm{e}-06$ & 2.28 & $8.33 e-01$ & $1.76 \mathrm{e}-01$ & 16.8 & 37.2 & 0.211 & 2.21 & $1.01 \mathrm{e}-07$ \\
\hline $7 \mathrm{H}-5,105-107$ & 62.55 & $4.29 \mathrm{e}-03$ & $4.05 \mathrm{e}-06$ & 3.11 & $5.26 \mathrm{e}-01$ & $1.21 \mathrm{e}-01$ & 18.8 & 40.4 & 0.230 & 2.15 & $4.18 \mathrm{e}-08$ \\
\hline $7 \mathrm{H}-6,61-63$ & 63.61 & $2.63 \mathrm{e}-03$ & $2.19 \mathrm{e}-06$ & 3.80 & $7.71 \mathrm{e}-01$ & $2.04 \mathrm{e}-01$ & 11.2 & 15.1 & 0.265 & 1.35 & $3.07 \mathrm{e}-07$ \\
\hline $8 \mathrm{H}-1,63-65$ & 65.63 & $6.91 \mathrm{e}-03$ & $5.31 \mathrm{e}-06$ & 2.57 & $6.95 \mathrm{e}-01$ & $1.56 \mathrm{e}-01$ & 17.1 & 36.6 & 0.224 & 2.14 & $-2.19 \mathrm{e}-08$ \\
\hline $8 \mathrm{H}-3,62-64$ & 68.62 & $5.23 \mathrm{e}-03$ & $3.55 \mathrm{e}-06$ & 2.12 & $3.90 \mathrm{e}-01$ & $8.95 \mathrm{e}-02$ & 17.4 & 38.4 & 0.229 & 2.21 & $2.56 \mathrm{e}-08$ \\
\hline $9 \mathrm{H}-2,91-93$ & 76.91 & $5.18 \mathrm{e}-03$ & $4.14 \mathrm{e}-06$ & 2.58 & $6.66 \mathrm{e}-01$ & $1.57 \mathrm{e}-01$ & 17.8 & 38.5 & 0.236 & 2.16 & $-2.56 \mathrm{e}-08$ \\
\hline $9 \mathrm{H}-3,43-45$ & 77.93 & $2.87 \mathrm{e}-03$ & $2.63 \mathrm{e}-06$ & 3.49 & $2.66 \mathrm{e}-01$ & $6.03 e-02$ & 18.0 & 39.0 & 0.227 & 2.17 & $6.77 \mathrm{e}-08$ \\
\hline $9 \mathrm{H}-6,20-22$ & 82.20 & $8.41 \mathrm{e}-03$ & $6.09 \mathrm{e}-06$ & 2.51 & $8.87 e-01$ & $1.92 \mathrm{e}-01$ & 16.5 & 35.9 & 0.216 & 2.18 & $8.98 \mathrm{e}-08$ \\
\hline $9 \mathrm{H}-6,109-111$ & 83.09 & $2.98 \mathrm{e}-03$ & $2.35 \mathrm{e}-06$ & 1.87 & $3.61 \mathrm{e}-01$ & $8.07 \mathrm{e}-02$ & 16.7 & 35.0 & 0.224 & 2.10 & $1.25 \mathrm{e}-07$ \\
\hline $10 \mathrm{H}-3,51-53$ & 87.51 & $7.57 \mathrm{e}-03$ & $6.08 \mathrm{e}-06$ & 3.08 & $8.89 \mathrm{e}-01$ & $2.00 \mathrm{e}-01$ & 16.9 & 36.5 & 0.225 & 2.16 & $4.70 \mathrm{e}-08$ \\
\hline $10 \mathrm{H}-3,87-89$ & 87.87 & $4.78 \mathrm{e}-03$ & $3.41 \mathrm{e}-06$ & 1.40 & $3.47 \mathrm{e}-01$ & $8.08 \mathrm{e}-02$ & 18.8 & 39.3 & 0.233 & 2.09 & $-2.84 \mathrm{e}-08$ \\
\hline $10 \mathrm{H}-4,89-91$ & 89.39 & $4.84 \mathrm{e}-03$ & $3.67 \mathrm{e}-06$ & 2.29 & $5.02 \mathrm{e}-01$ & $1.11 \mathrm{e}-01$ & 16.8 & 34.7 & 0.221 & 2.07 & $4.46 \mathrm{e}-08$ \\
\hline $10 \mathrm{H}-5,100-102$ & 91.00 & $4.43 e-03$ & $3.12 \mathrm{e}-06$ & 0.75 & $1.11 \mathrm{e}+00$ & $1.54 \mathrm{e}-01$ & 13.0 & 34.1 & 0.139 & 2.62 & $-4.61 \mathrm{e}-08$ \\
\hline $10 \mathrm{H}-6,55-57$ & 92.05 & $4.93 \mathrm{e}-03$ & $3.96 \mathrm{e}-06$ & 2.70 & $4.84 \mathrm{e}-01$ & $8.75 \mathrm{e}-02$ & 15.4 & 33.7 & 0.181 & 2.19 & $2.29 \mathrm{e}-08$ \\
\hline \multicolumn{12}{|l|}{ 152-919B- } \\
\hline $3 \mathrm{H}-3,92-94$ & 93.92 & $3.80 \mathrm{e}-03$ & $3.08 \mathrm{e}-06$ & 1.75 & $5.85 \mathrm{e}-01$ & $8.36 \mathrm{e}-02$ & 12.5 & 34.9 & 0.143 & 2.79 & $-2.09 e-08$ \\
\hline $3 \mathrm{H}-5,51-53$ & 96.51 & $4.90 \mathrm{e}-03$ & $4.01 \mathrm{e}-06$ & 3.40 & $6.92 \mathrm{e}-01$ & $1.37 \mathrm{e}-01$ & 15.4 & 33.0 & 0.198 & 2.14 & $1.73 \mathrm{e}-08$ \\
\hline $3 \mathrm{H}-6,83-85$ & 98.33 & $6.09 \mathrm{e}-03$ & $4.60 \mathrm{e}-06$ & 2.55 & $6.89 \mathrm{e}-01$ & $1.43 \mathrm{e}-01$ & 16.5 & 35.8 & 0.208 & 2.17 & $4.44 \mathrm{e}-09$ \\
\hline $3 \mathrm{H}-7,45-47$ & 99.45 & $4.99 \mathrm{e}-03$ & $4.00 \mathrm{e}-06$ & 3.12 & $3.45 \mathrm{e}-01$ & $6.01 \mathrm{e}-02$ & 15.1 & 34.1 & 0.174 & 2.26 & $7.13 e-08$ \\
\hline $4 \mathrm{H}-1,101-103$ & 100.51 & $2.82 \mathrm{e}-03$ & $2.83 \mathrm{e}-06$ & 2.76 & $2.78 \mathrm{e}-01$ & $5.27 \mathrm{e}-02$ & 15.9 & 34.6 & 0.190 & 2.18 & $5.92 \mathrm{e}-08$ \\
\hline $4 \mathrm{H}-2,36-38$ & 101.36 & $8.64 \mathrm{e}-03$ & $6.30 \mathrm{e}-06$ & 1.80 & $8.84 \mathrm{e}-01$ & $1.62 \mathrm{e}-01$ & 15.1 & 34.1 & 0.183 & 2.26 & $6.12 \mathrm{e}-08$ \\
\hline $4 \mathrm{H}-3,50-52$ & 103.00 & $3.98 \mathrm{e}-03$ & $3.28 \mathrm{e}-06$ & 3.07 & $5.07 \mathrm{e}-01$ & $1.20 \mathrm{e}-01$ & 17.7 & 37.4 & 0.237 & 2.11 & $9.65 e-08$ \\
\hline $4 \mathrm{H}-5,16-18$ & 105.66 & $6.13 e-03$ & $4.32 \mathrm{e}-06$ & 1.45 & $6.67 e-01$ & $1.19 \mathrm{e}-01$ & 14.4 & 32.9 & 0.178 & 2.28 & $5.10 \mathrm{e}-08$ \\
\hline $4 \mathrm{H}-6,89-91$ & 107.89 & $2.87 \mathrm{e}-03$ & $2.37 \mathrm{e}-06$ & 3.88 & $2.95 \mathrm{e}-01$ & $5.77 \mathrm{e}-02$ & 16.1 & 35.8 & 0.196 & 2.22 & $-3.00 \mathrm{e}-08$ \\
\hline $5 \mathrm{H}-2,9-11$ & 110.59 & $7.33 \mathrm{e}-03$ & $5.03 \mathrm{e}-06$ & 1.82 & $6.71 \mathrm{e}-01$ & $1.44 \mathrm{e}-01$ & 17.2 & 37.0 & 0.215 & 2.15 & $-3.74 \mathrm{e}-08$ \\
\hline $5 \mathrm{H}-3,105-107$ & 113.05 & $5.82 \mathrm{e}-03$ & $4.70 \mathrm{e}-06$ & 3.05 & $5.61 \mathrm{e}-01$ & $1.34 \mathrm{e}-01$ & 17.8 & 37.8 & 0.239 & 2.12 & $8.63 e-08$ \\
\hline $5 \mathrm{H}-4,35-37$ & 113.85 & $3.51 \mathrm{e}-03$ & $3.01 \mathrm{e}-06$ & 3.80 & $4.47 \mathrm{e}-01$ & $1.11 \mathrm{e}-01$ & 18.2 & 38.5 & 0.248 & 2.12 & $-2.01 \mathrm{e}-08$ \\
\hline $5 \mathrm{H}-5,11-13$ & 115.11 & $3.14 \mathrm{e}-03$ & $3.03 \mathrm{e}-06$ & 3.53 & $1.09 \mathrm{e}-01$ & $1.76 \mathrm{e}-02$ & 13.2 & 33.3 & 0.161 & 2.52 & $-9.51 \mathrm{e}-09$ \\
\hline $6 \mathrm{H}-1,67-69$ & 119.17 & $4.48 \mathrm{e}-03$ & $4.10 \mathrm{e}-06$ & 2.73 & $5.58 \mathrm{e}-01$ & $1.23 \mathrm{e}-01$ & 17.5 & 38.6 & 0.220 & 2.21 & $9.05 e-08$ \\
\hline $6 \mathrm{H}-2,114-116$ & 121.14 & $6.04 \mathrm{e}-03$ & $5.03 \mathrm{e}-06$ & 1.47 & $8.30 \mathrm{e}-01$ & $1.60 \mathrm{e}-01$ & 15.5 & 35.8 & 0.193 & 2.31 & $-1.38 \mathrm{e}-08$ \\
\hline $6 \mathrm{H}-3,80-82$ & 122.30 & $4.96 \mathrm{e}-03$ & $3.65 \mathrm{e}-06$ & 3.14 & $4.80 \mathrm{e}-01$ & $9.16 \mathrm{e}-02$ & 16.4 & 36.4 & 0.191 & 2.22 & $5.64 \mathrm{e}-08$ \\
\hline $6 \mathrm{H}-5,57-59$ & 125.07 & $8.39 \mathrm{e}-03$ & $5.80 \mathrm{e}-06$ & 2.12 & $7.70 \mathrm{e}-01$ & $1.75 \mathrm{e}-01$ & 16.9 & 36.4 & 0.227 & 2.15 & $1.12 \mathrm{e}-07$ \\
\hline $6 \mathrm{H}-6,137-139$ & 127.37 & $5.18 \mathrm{e}-03$ & $4.28 \mathrm{e}-06$ & 2.36 & $5.39 \mathrm{e}-01$ & $1.12 \mathrm{e}-01$ & 15.6 & 37.9 & 0.208 & 2.43 & $7.62 \mathrm{e}-08$ \\
\hline $7 \mathrm{H}-3,81-83$ & 131.81 & $4.16 \mathrm{e}-03$ & $3.03 e-06$ & 2.67 & $4.01 \mathrm{e}-01$ & $6.46 \mathrm{e}-02$ & 14.5 & 33.5 & 0.161 & 2.31 & $8.88 \mathrm{e}-08$ \\
\hline $7 \mathrm{H}-5,61-63$ & 134.61 & $5.70 \mathrm{e}-03$ & $3.75 \mathrm{e}-06$ & 2.34 & $6.04 \mathrm{e}-01$ & $9.09 \mathrm{e}-02$ & 13.5 & 33.4 & 0.150 & 2.47 & $1.24 \mathrm{e}-08$ \\
\hline $7 \mathrm{H}-5,113-115$ & 135.13 & $8.21 \mathrm{e}-03$ & $5.57 \mathrm{e}-06$ & 2.44 & $8.10 \mathrm{e}-01$ & $1.73 \mathrm{e}-01$ & 16.6 & 35.3 & 0.214 & 2.13 & $1.13 \mathrm{e}-07$ \\
\hline $7 \mathrm{H}-7,18-20$ & 137.18 & $5.63 \mathrm{e}-03$ & $3.46 \mathrm{e}-06$ & 2.17 & $7.46 \mathrm{e}-01$ & $1.51 \mathrm{e}-01$ & 16.3 & 35.7 & 0.202 & 2.19 & $4.15 \mathrm{e}-08$ \\
\hline $8 \mathrm{H}-2,98-100$ & 139.98 & $5.18 \mathrm{e}-03$ & $3.60 \mathrm{e}-06$ & 1.72 & $5.91 \mathrm{e}-01$ & $1.18 \mathrm{e}-01$ & 15.7 & 35.8 & 0.200 & 2.28 & $7.24 \mathrm{e}-09$ \\
\hline $8 \mathrm{H}-3,51-53$ & 141.01 & $4.01 \mathrm{e}-03$ & $3.30 \mathrm{e}-06$ & 2.77 & $4.34 \mathrm{e}-01$ & $8.68 \mathrm{e}-02$ & 16.2 & 36.1 & 0.200 & 2.23 & $1.65 \mathrm{e}-08$ \\
\hline $8 \mathrm{H}-4,114-116$ & 143.14 & $5.40 \mathrm{e}-03$ & $5.10 \mathrm{e}-06$ & 2.26 & $8.11 \mathrm{e}-01$ & $2.00 \mathrm{e}-01$ & 18.5 & 38.3 & 0.247 & 2.07 & $5.29 \mathrm{e}-08$ \\
\hline $8 \mathrm{H}-6,63-65$ & 145.63 & $4.14 \mathrm{e}-03$ & $3.20 \mathrm{e}-06$ & 2.14 & $7.51 \mathrm{e}-01$ & $1.50 \mathrm{e}-01$ & 15.7 & 33.9 & 0.200 & 2.16 & $5.45 \mathrm{e}-08$ \\
\hline $8 \mathrm{H}-6,88-90$ & 145.88 & $4.61 \mathrm{e}-03$ & $3.59 \mathrm{e}-06$ & 4.10 & $4.46 \mathrm{e}-01$ & $8.58 \mathrm{e}-02$ & 16.1 & 35.8 & 0.192 & 2.22 & $7.99 \mathrm{e}-08$ \\
\hline
\end{tabular}

Notes: $\kappa_{\mathrm{lf}}=$ low-field volume susceptibility, $\chi_{\mathrm{lf}}=$ low-field mass susceptibility, $\kappa_{\mathrm{fd}}=$ frequency dependence of susceptibility, $\mathrm{J}_{\mathrm{s}}=$ saturation magnetization, $\mathrm{J}_{\mathrm{r}}=$ saturation remanence,

$\mathrm{H}_{\mathrm{c}}=$ coercivity, $\mathrm{H}_{\mathrm{cr}}=$ coercivity of remanence, and $\chi_{\mathrm{hf}}=$ high-field mass susceptibility.

\section{VOLCANIC ROCK RESULTS}

The basaltic lavas from Hole 917A are entirely subaerial flows that largely vary in thickness, suggesting that oxidation state and cooling rate are quite variable in the sequence. Figure 7, which was plotted at every 10th point of the acquired data as in Figure 1, shows a downhole variation of magnetic susceptibility for the lava flow sequence. The values primarily range from $2 \times 10^{-3}$ to $20 \times 10^{-3} \mathrm{SI}$. Values lower than $2 \times 10^{-3} \mathrm{SI}$ are caused by voids between rock pieces. Although the magnetic susceptibility is quite variable in finer scale, the values grossly correlate to the three stratigraphic series: the baseline value $\left(\sim 4 \times 10^{-3} \mathrm{SI}\right)$ for the Middle Series (evolved basalts and dacites $)$ is higher than those of the Upper $\left(\sim 2 \times 10^{-3}\right.$ SI, olivine basalts and picrites) and Lower Series $\left(\sim 2 \times 10^{-3}\right.$ SI, basalts). Presumably, the baseline value for each series reflects the susceptibility of the less oxidized massive central portion of lava flow. Within the Upper Series lavas, which are predominantly olivine basalts and picrites, low susceptibility values occur at the interval of picrites (150-175 mbsf). These facts suggest that the susceptibility of less oxidized basalt depends on the rock chemistry controlling the concentration of original titanomagnetite. Dacites (345-375 mbsf) are characterized by low susceptibility $\left(<10 \times 10^{-3} \mathrm{SI}\right)$ as expected. There are highs in the
Lower Series at just above 400 and $500 \mathrm{mbsf}$, and at the interval of 560-590 mbsf. The former two highs correspond to the portions of scoriaceous breccias and the latter to the interval of fractured and brecciated lavas including mylonites and faulted contacts.

An example of fine-scale variation reflecting lava successions can be seen in Core 152-917A-13R (Fig. 8). The baseline, which is representative of the inner massive part of lava flows, is at $\sim 4 \times 10^{-3} \mathrm{SI}$ through the three igneous units. A prominent peak $\left(>40 \times 10^{-3} \mathrm{SI}\right)$ in the upper part of Unit 17 (104.4-105.4 mbsf) corresponds to black and finely porous scoriaceous breccia. A relatively small and narrow peak at the uppermost of Unit 18 occurs at a red and porous portion, which is commonly observed at the top of lava flows through the recovered lava piles. However, the flow top of Unit 17, which is finely porous scoriaceous breccia like the high-susceptibility portion (104.4-105.4 mbsf) but has a reddish color and a more intensely oxidized feature than that of Unit 18, does not exhibit a peak in susceptibility variation.

Figure 9A and 9B show the two common types of thermomagnetic curves of volcanic rocks from Hole 917A. Almost reversible curves between heating and cooling runs and Curie point of $580^{\circ} \mathrm{C}$ are indicative of pure magnetite (Fig. 9A). This type of curve is most common throughout the Upper, Middle, and Lower Series. Another 
Holes 918A, 918B and 918C (lithologic Unit I)
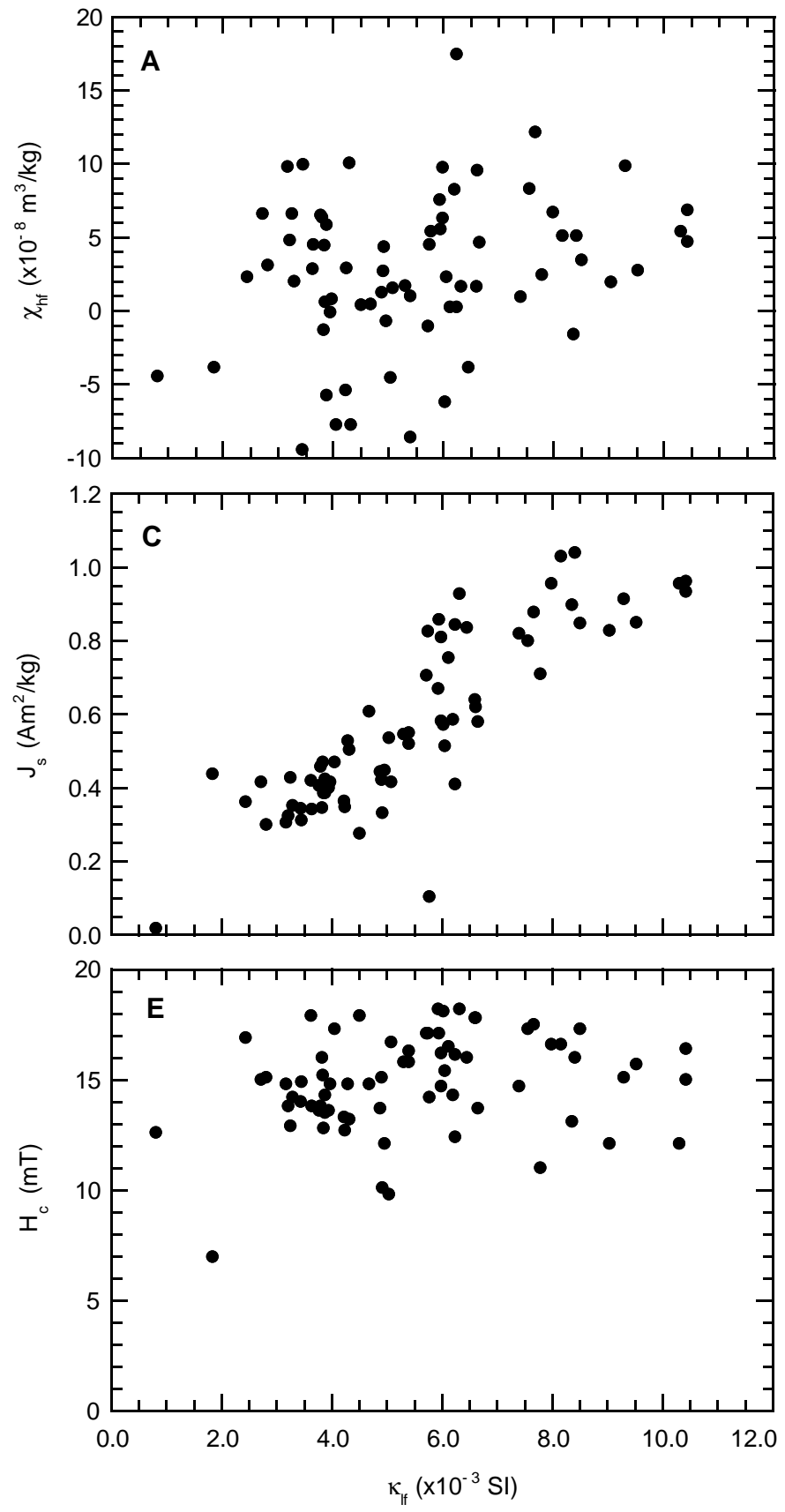

Holes 919A and 919B
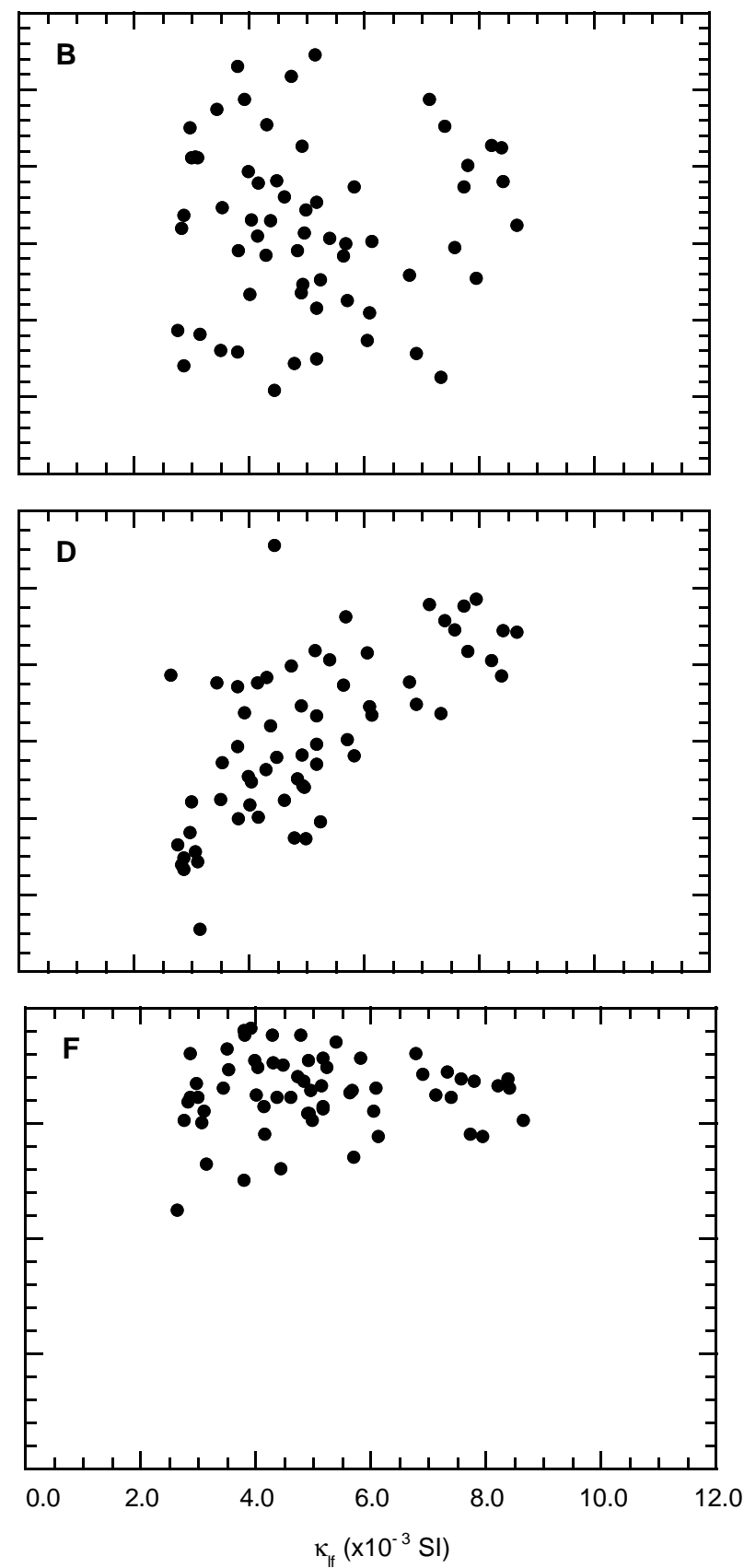

Figure 6. Hysteresis parameters plotted against low-field volume susceptibility $\left(\kappa_{\mathrm{lf}}\right)$ for sediment discrete samples from Holes 918A, 918B, 918C, 919A, and 919B. High-field mass susceptibility $\left(\chi_{\mathrm{lf}}\right)$ vs. $\kappa_{\mathrm{lf}}$ for Sites 918 (A) and $919(\mathbf{B})$, saturation magnetization $\left(\mathrm{J}_{\mathrm{s}}\right)$ vs. $\kappa_{\mathrm{lf}}$ for Sites 918 (C) and 919 (D), and coercivity $\left(\mathrm{H}_{\mathrm{c}}\right)$ vs. $\mathrm{K}_{\mathrm{lf}}$ for Sites $918(\mathbf{E})$ and $919(\mathbf{F})$.

minor but ubiquitously occurring type of thermomagnetic curve documents the presence of titanomaghemite (Fig. 9B). The samples containing titanomaghemite are marked by the low magnetic stability (the median destructive fields of anhysteretic remanent magnetization [MDF of ARM] are less than 10 mT [Larsen et al., 1994; Larsen, Saunders, Clift, et al., 1994], whereas the samples with magnetite exhibit very high magnetic stability [MDF of ARM > $20 \mathrm{mT}]$ ). Such a high stability of magnetite-bearing samples is probably caused by the exsolution of titanomagnetite at deuteric oxidation. In spite of contrasting magnetic mineralogy and stability, the values of susceptibility are not as different as between magnetite- and titanomaghemitebearing samples (Larsen, Saunders, Clift, et al., 1994).

Scoriaceous breccia samples have characteristic thermomagnetic curves. The example shown in Figure $9 \mathrm{C}$ is taken from the susceptibility peak in the upper part of Unit 17 (Fig. 8); the measured susceptibility on this discrete sample was $2.78 \times 10^{-2}$ SI. In addition to a 
Hole 917A

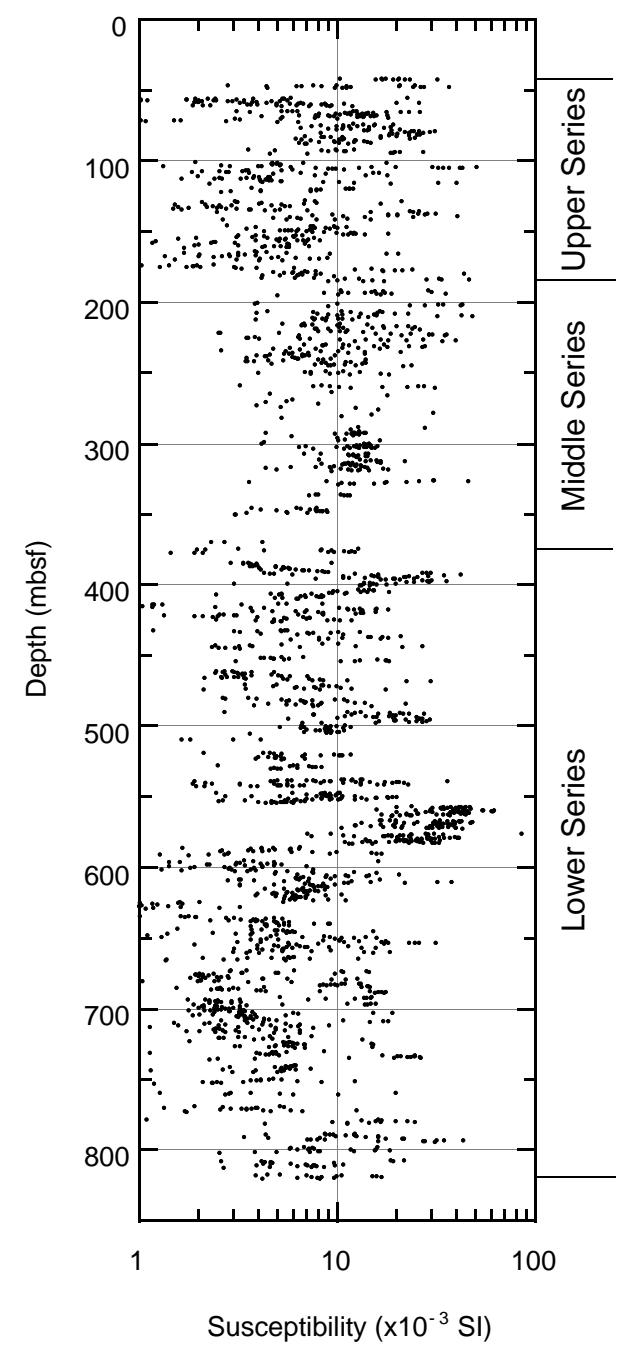

Figure 7. Downhole variation of whole-core magnetic susceptibility of volcanic rocks from Hole 917A. Susceptibility data points are plotted at every 10th point of acquired data to avoid confusion.

kink at about $580^{\circ} \mathrm{C}$, which is indicative of nearly pure magnetite, there is another kink at about $300^{\circ} \mathrm{C}$. Because the cooling curve is not far above the heating curve, I discard the possibility of titanomaghemite. It is probable that Ti-rich titanomagnetite coexists with nearly pure magnetite and forms double kinks. Ti-rich titanomagnetite may cause the susceptibility enhancement in scoriaceous breccia portions as found at just above 400 and 500 mbsf at Hole 917A (Fig. 7).

Figure 9D shows a representative thermomagnetic curve for a sample taken from an oxidized reddish top of a lava flow. A major kink at about $580^{\circ} \mathrm{C}$ is indicative of nearly pure magnetite, and there is another slight kink above $650^{\circ} \mathrm{C}$ of hematite. The cooling curve is far above the heating curve in the argon gas atmosphere, which is probably due to reduction of hematite or iron-bearing silicates into magnetite. Hematite is thought to be a product of oxidation of magnetite at the top of a lava flow through a process like a martitization often observed in red beds (e.g., Walker et al., 1981). Because saturation magnetization of hematite is lower than that of magnetite by two orders of magnitude, oxidation of magnetite into hematite effectively reduces the magnetic susceptibility. The reason that no visible
Core 152-917A-13R

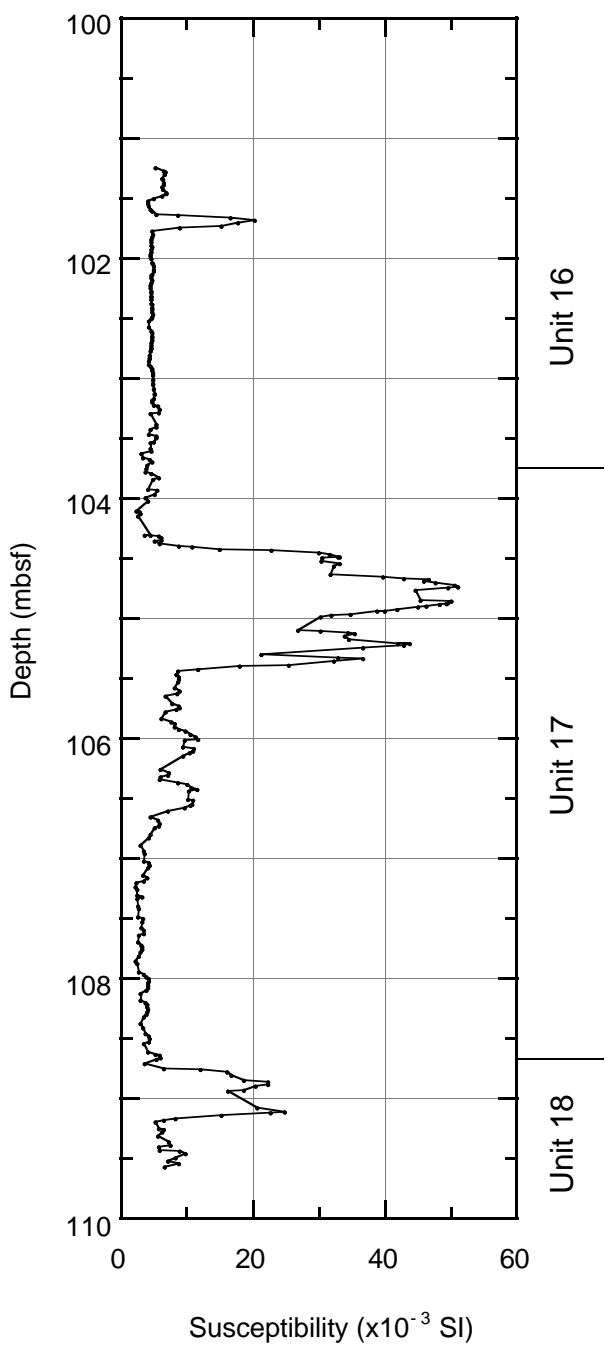

Figure 8 . Whole-core magnetic susceptibility variation for volcanic rocks from Core 152-917A-13R.

peak can be seen at the top of Unit 17 (intensely oxidized scoriaceous breccias, Fig. 8) is probably that the decrease of susceptibility due to the oxidation of magnetite into hematite compensated for the increase of susceptibility in porous scoriaceous breccias.

\section{SUMMARY}

Some applications of whole-core magnetic susceptibility data were presented for sediments and volcanic rocks recovered during Leg 152. The origin of the susceptibility signal was discussed on the basis of rock magnetic data obtained on shore, not by just comparing the susceptibility variation with lithologic data.

The gross feature of susceptibility variation for sediments at Site 918 revealed the variation of detrital input. The susceptibility is negatively correlated to the carbonate content. At the upper part of lithologic Unit II (nannofossil chalk and volcanic silt), the susceptibility gradually increases upward associated with fine-scale oscillations. This trend suggests that detrital input gradually increased far below the bottom of glaciomarine sediment (lithologic Unit I). The finding could not be revealed by measurements on discrete samples or visual 

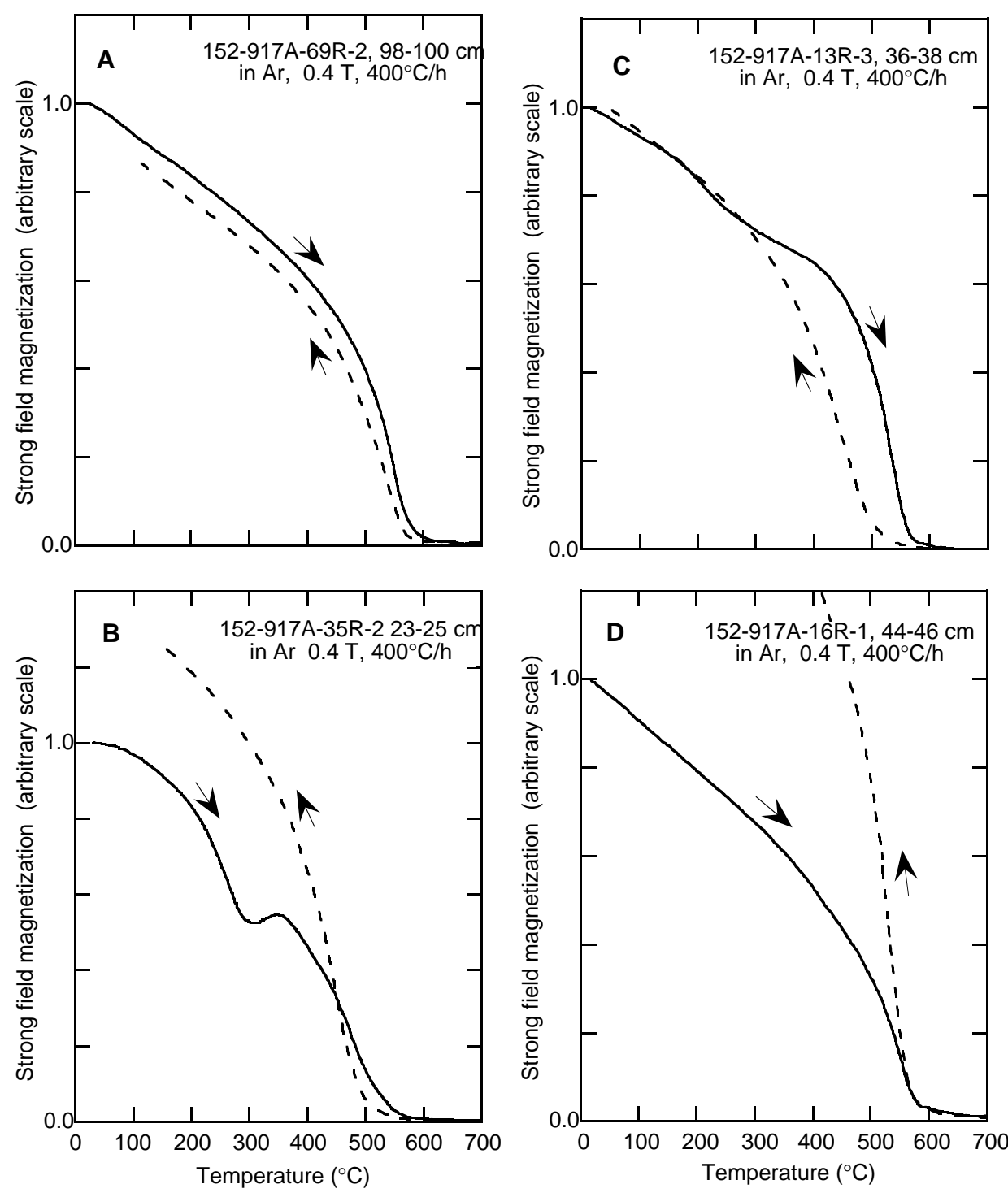

Figure 9. Representative thermomagnetic curves for volcanic rocks from Hole 917A. A. A magnetite-bearing rock. B. A titanomaghemite-bearing rock. C. A scoriaceous breccia. D. Oxidized reddish top of a lava flow.

core descriptions. If combined with GRAPE density measurements, the whole-core susceptibility log can detect the rock type of a dropstone as well as its position. By correlating the susceptibility variation of the overlapping cores obtained from Site 919, it was possible to detect a slightly different sedimentation rate and missing intervals at core break. Thermomagnetic data indicated that the major carrier of susceptibility signal is nearly pure magnetite, but some minor iron sulfides might be present. Based on the hysteresis parameters, I confirm that the susceptibility depends primarily on the concentration of ferromagnetic minerals, the contribution of para- and diamagnetic material is negligible, and the grain size of ferromagnetic minerals does not affect susceptibility significantly.

For volcanic rocks, the susceptibility variation is influenced by the original rock chemistry in large scale and the degree of oxidation in small scale. The less oxidized inner part of lava flows, which contain titanomaghemite or exsolved titanomagnetite, forms the baseline for the susceptibility variation, and the baseline values depend on the degree of evolution of basaltic rocks. The most evolved basalts in the Middle Series have the highest baseline values, the least evolved picrites have the lowest, and intermediate basalts of the Lower Series have the intermediate baseline values. This gross variation reflects the concentration of titanomagnetite depending on the rock chemistry. Locally scoriaceous breccia and fractured rocks are associated with susceptibility highs. The scoriaceous brecciated portion that frequently occurs near the top of a lava flow exhibits a susceptibility peak that is associated with the formation of titanomagnetite. Although some lava flow tops are marked by susceptibility enhancement, very intense oxidation at other tops reduces the susceptibility by oxidizing magnetite into hematite. Although not available for Leg 152 , in situ susceptibility logging into subaerial volcanic sequences such as seaward-dipping reflector sequences will be useful on future ODP legs to take a continuous profile of lava flow succession.

\section{ACKNOWLEDGMENTS}

Whole-core susceptibility measurements during Leg 152 were primarily done by T. Kimura. I am extremely grateful to him for his invaluable efforts. I would like to thank M. Torii at Kyoto University and D.J. Dunlop at the University of Toronto for the use of their fa- 
cilities. I am also grateful to N. Nemoto, who helped me with susceptibility measurements on discrete samples. This research was supported by a Fellowship of the Japan Society for the Promotion of Science for Japanese Junior Scientists and a Grant-in-aid for scientific research of the Ministry of Education of Japan (No. 04649702).

\section{REFERENCES}

Bloemendal, J., and deMenocal, P., 1989. Evidence for a change in the periodicity of tropical climate cycles at $2.4 \mathrm{Myr}$ from whole-core magnetic susceptibility measurements. Nature, 342:897-900.

deMenocal, P., Bloemendal, J., and King, J., 1991. A rock-magnetic record of monsoonal dust deposition to the Arabian Sea: evidence for a shift in the mode of deposition at 2.4 Ma. In Prell, W.L., Niitsuma, N., et al., Proc. ODP, Sci. Results, 117: College Station, TX (Ocean Drilling Program), 389-407.

Heider, F., Zitzelsberger, A., and Fabian, K., 1994. Magnetic susceptibility of grown magnetite crystals from $0.1 \mu \mathrm{m}$ to $6 \mathrm{~mm}$. Eos, 75:187.

Karlin, R., and Levi, S., 1983. Diagenesis of magnetic minerals in recent hemipelagic sediments. Nature, 303:327-330.

Larsen, H.C., Saunders, A.D., Clift, P.D., et al., 1994. Proc. ODP, Init. Repts., 152: College Station, TX (Ocean Drilling Program).

Larsen, H.C., Saunders, A.D., Clift, P.D., Beget, J., Wei, W., Spezzaferri, S., and the ODP Leg 152 Scientific Party, 1994. Seven million years of glaciation in Greenland. Science, 264:952-955.
Leg 154 Scientific Party, 1995. Ceara Rise sediments document ancient climate change. Eos, 76:41-45.

Maher, B.A., 1988. Magnetic properties of some synthetic sub-micron magnetites. Geophys. J. R. Astron. Soc., 94:83-96.

Moskowitz, B.M., 1995. Fundamental physical constants and conversion factors. In Ahrens, T.J. (Eds.), Global Earth Physics: A Handbook of Physical Constants. Am. Geophys. Union, 346-355.

Roberts, A.P., 1995. Magnetic properties of sedimentary greigite $\left(\mathrm{Fe}_{3} \mathrm{~S}_{4}\right)$. Earth Planet. Sci. Lett., 134:227-236.

Robinson, S.G., 1990. Applications for whole-core magnetic susceptibility measurements of deep-sea sediments: Leg 115 results. In Duncan, R.A., Backman, J., Peterson, L.C., et al., Proc. ODP, Sci. Results, 115: College Station, TX (Ocean Drilling Program), 737-771.

Schwarz, E.J., 1975. Magnetic properties of pyrrhotite and their use in applied geology and geophysics. Pap.-Geol. Surv. Can., 74-59:1-24.

Walker, T.R., Larson, E.E., and Hoblitt, R.P., 1981. The nature and origin of hematite in the Moenkopi Formation (Triassic), Colorado Plateau: a contribution to the origin of magnetism in red beds. J. Geophys. Res., 86:317-333.

Date of initial receipt: 10 August 1995

Date of acceptance: 23 May 1996

Ms 152SR-225 